\title{
Single-epoch RTK performance assessment of tightly combined BDS- 2 and newly complete BDS-3
}

\author{
Wanke Liu', Mingkui Wu'², Xiaohong Zhang ${ }^{1 *} \mathbb{0}$, Wang Wang ${ }^{1}$, Wei Ke ${ }^{1}$ and Zhiqin Zhu ${ }^{1}$
}

\begin{abstract}
The BeiDou global navigation satellite system (BDS-3) constellation deployment has been completed on June 23, 2020, with a full constellation comprising 30 satellites. In this study, we present the performance assessment of singleepoch Real-Time Kinematic (RTK) positioning with tightly combined BeiDou regional navigation satellite system (BDS-2) and BDS-3. We first investigate whether code and phase Differential Inter-System Biases (DISBs) exist between the legacy B1//B3I signals of BDS-3/BDS-2. It is discovered that the DISBs are in fact about zero for the baselines with the same or different receiver types at their endpoints. These results imply that BDS-3 and BDS-2 are fully interoperable and can be regarded as one constellation without additional DISBs when the legacy B1//B3I signals are used for precise relative positioning. Then we preliminarily evaluate the single-epoch short baseline RTK performance of tightly combined BDS-2 and the newly completed BDS-3. The performance is evaluated through ambiguity resolution success rate, ambiguity dilution of precision, as well as positioning accuracy in kinematic and static modes using the datasets collected in Wuhan. Experimental results demonstrate that the current BDS-3 only solutions can deliver comparable ambiguity resolution performance and much better positioning accuracy with respect to BDS-2 only solutions. Moreover, the RTK performance is much improved with tightly combined BDS-3/BDS-2, particularly in challenging or harsh conditions. The single-frequency single-epoch tightly combined BDS-3/BDS-2 solution could deliver an ambiguity resolution success rate of $96.9 \%$ even with an elevation cut-off angle of $40^{\circ}$, indicating that the tightly combined BDS-3/BDS-2 could achieve superior RTK positioning performance in the Asia-Pacific region. Meanwhile, the three-dimensional (East/North/Up) positioning accuracy of BDS-3 only solution $(0.52 \mathrm{~cm} / 0.39 \mathrm{~cm} / 2.14 \mathrm{~cm})$ in the kinematic test is significantly better than that of the BDS-2 only solution $(0.85 \mathrm{~cm} / 1.02 \mathrm{~cm} / 3.01 \mathrm{~cm})$ due to the better geometry of the current BDS-3 constellation. The tightly combined BDS-3/BDS-2 solution can provide the positioning accuracy of $0.52 \mathrm{~cm}, 0.22 \mathrm{~cm}$, and $1.80 \mathrm{~cm}$, respectively.
\end{abstract}

Keywords: BeiDou global navigation satellite system, BDS-2, Real-time kinematic, Differential inter-system bias, Ambiguity resolution

\section{Introduction}

BeiDou Navigation Satellite System (BDS) was developed into three phases, the BeiDou demonstration navigation satellite system (BDS-1), the BeiDou regional navigation satellite system (BDS-2), and the BeiDou

\footnotetext{
*Correspondence: xhzhang@sgg.whu.edu.cn

${ }^{1}$ School of Geodesy and Geomatics, Wuhan University, Wuhan 430079,

China

Full list of author information is available at the end of the article
}

global navigation satellite system (BDS-3) (Yang et al. 2019b). The built-up of BDS-3 began with the launch of five experimental satellites from March 2015 to February 2016, including three Medium Earth Orbit (MEO) and two Inclined Geostationary Orbit (IGSO) satellites, which were designed to perform in-orbit verification of the new payloads, new signals, and new techniques (Xie et al. 2018; Yang et al. 2018, 2019b). The operational BDS-3 was started in November 2017, and in the end of 2018, the BDS-3 primary system consisting of $18 \mathrm{MEO}$ 
satellites was completed and began to provide services to global users with at least five visible satellites (Yang et al. 2020). By the end of 2019, the core BDS-3 constellation comprising $24 \mathrm{MEO}$ satellites was completed, which comprehensively promoted the system service worldwide (China Satellite Navigation Office 2019). On June 23, 2020, the last BDS-3 Geostationary Orbit (GEO) satellite was successfully launched, signifying the accomplishment of the BDS-3 constellation deployment, including $24 \mathrm{MEO}$, three IGSO, and three GEO satellites. As of July 2020, the constellation status of BDS-3 is listed in Table 1 (CSNO-TARC 2020). The BDS-3 operational satellites transmit multiple open service navigation signals on five different frequency bands, i.e., the legacy B1I (1561.098 MHz) and B3I (1268.52 MHz) signals, the new B1C (1575.42 MHz), B2a (1176.45 MHz), and B2b (1207.14 MHz) signals that having the same center frequency with Global Positioning System (GPS) L1/L5 as well as Galileo Navigation Satellite System (Galileo) E1/E5a/E5b signals (Lu et al. 2019; Yang et al. 2019b).

After the BDS-3 demonstration system with five experimental satellites was established, the observational quality of its new navigation signals was intensively investigated in aspects of the carrier-to-noise power density ratio, code multipath effect, triple-frequency carrier phase combination, as well as carrier phase and code observation precision, etc. (Xie et al. 2017; Zhang et al. 2017, 2019a; Zhou et al. 2018). It was proved that the data quality of B1C/B2a/B2b observations of BDS-3 demonstration system was comparable to those of GPS and Galileo. Meanwhile, its contribution to the precise positioning was evaluated by several researchers. The results revealed that the additional observations from BDS-3 demonstration system improved the precise positioning

Table 1 Constellation status of BDS-3 as of July 2020

\begin{tabular}{|c|c|c|c|c|c|}
\hline PRN code & Common name & Date of launch & Orbit & Status & $\begin{array}{l}\text { Fundamental PNT } \\
\text { service signal }\end{array}$ \\
\hline $\mathrm{C} 19$ & BDS-3 MEO-1 & 5.11 .2017 & MEO & Operational & $\mathrm{B} 1 \mathrm{l} / \mathrm{B} 1 \mathrm{C} / \mathrm{B} 2 \mathrm{a} / \mathrm{B} 2 \mathrm{~b} / \mathrm{B} 3 \mathrm{I}$ \\
\hline $\mathrm{C} 20$ & BDS-3 MEO-2 & 5.11 .2017 & MEO & Operational & \\
\hline $\mathrm{C} 27$ & BDS-3 MEO-7 & 11.01 .2018 & MEO & Operational & \\
\hline $\mathrm{C} 28$ & BDS-3 MEO-8 & 11.01 .2018 & MEO & Operational & \\
\hline $\mathrm{C} 22$ & BDS-3 MEO-4 & 12.02 .2018 & MEO & Operational & \\
\hline $\mathrm{C} 21$ & BDS-3 MEO-3 & 12.02 .2018 & MEO & Operational & \\
\hline C29 & BDS-3 MEO-9 & 29.03.2018 & MEO & Operational & \\
\hline $\mathrm{C} 30$ & BDS-3 MEO-10 & $29.03,2018$ & MEO & Operational & \\
\hline $\mathrm{C} 23$ & BDS-3 MEO-5 & 29.07.2018 & MEO & Operational & \\
\hline $\mathrm{C} 24$ & BDS-3 MEO-6 & 29.07.2018 & MEO & Operational & \\
\hline $\mathrm{C} 26$ & BDS-3 MEO-11 & 24.08 .2018 & MEO & Operational & \\
\hline $\mathrm{C} 25$ & BDS-3 MEO-12 & 24.08.2018 & MEO & Operational & \\
\hline $\mathrm{C} 32$ & BDS-3 MEO-13 & 19.09.2018 & MEO & Operational & \\
\hline $\mathrm{C} 33$ & BDS-3 MEO-14 & 19.09.2018 & MEO & Operational & \\
\hline $\mathrm{C} 35$ & BDS-3 MEO-16 & 15.10 .2018 & MEO & Operational & \\
\hline C34 & BDS-3 MEO-15 & 15.10 .2018 & MEO & Operational & \\
\hline $\mathrm{C} 36$ & BDS-3 MEO-17 & 18.11.2018 & MEO & Operational & \\
\hline C37 & BDS-3 MEO-18 & 18.11.2018 & MEO & Operational & \\
\hline C38 & BDS-3 IGSO-1 & 20.04 .2019 & IGSO & Operational & \\
\hline C39 & BDS-3 IGSO-2 & 25.06.2019 & IGSO & Operational & \\
\hline $\mathrm{C} 46$ & BDS-3 MEO-24 & 23.09.2019 & MEO & Operational & \\
\hline C45 & BDS-3 MEO-23 & 23.09.2019 & MEO & Operational & \\
\hline $\mathrm{C} 40$ & BDS-3 IGSO-3 & 5.11 .2019 & IGSO & Operational & \\
\hline $\mathrm{C} 44$ & BDS-3 MEO-22 & 23.11 .2019 & MEO & Operational & \\
\hline $\mathrm{C} 43$ & BDS-3 MEO-21 & 23.11 .2019 & MEO & Operational & \\
\hline $\mathrm{C} 41$ & BDS-3 MEO-19 & 16.12 .2019 & MEO & Operational & \\
\hline $\mathrm{C} 42$ & BDS-3 MEO-20 & 16.12.2019 & MEO & Operational & \\
\hline C59 & BDS-3 GEO-1 & 1.11.2018 & GEO & Operational & B11/B31 \\
\hline $\mathrm{C} 60$ & BDS-3 GEO-2 & 9.03 .2020 & GEO & Operational & \\
\hline $\mathrm{C} 61$ & BDS-3 GEO-3 & 23.06 .2020 & GEO & Experiment & \\
\hline
\end{tabular}


compared with BDS-2 only positioning (Zhang et al. 2017, 2018; Xu et al. 2018; Qu et al. 2019).

During the construction of operational BDS-3 constellation, several studies examined the data quality of B1C/ $\mathrm{B} 2 \mathrm{a} / \mathrm{B} 2 \mathrm{~b}$ observations of available BDS-3 satellites as of their writing and the results were similar to those of the experimental satellites (Xie et al. 2018; Wu et al. 2019a; $\mathrm{Xu}$ et al. 2019; Yang et al. 2019a). Regarding Real-Time Kinematic (RTK) positioning, initial assessments were presented recently based on the 18 new satellites and their navigation signals of the BDS-3 primary system. Zhang et al. (2019b) evaluated the RTK performance of combined BDS-3/BDS-2 solution using dual-frequency B1I/B3I observations with respect to BDS-2 only solution. It was demonstrated that additional BDS-3 observations could lead to improved RTK positioning accuracy in horizontal and vertical directions of the involved baseline vectors, with improvement by $1-2 \mathrm{~mm}$ for a $20 \mathrm{~m}$ baseline and by $1-2 \mathrm{~cm}$ for a $10 \mathrm{~km}$ baseline, respectively. Zhang et al. (2019c) indicated that the ambiguity resolution success rate of RTK was improved from 88.5 to 91.4\% by incorporating BDS-3 observations, whereas the positioning accuracy was comparable. Hou et al. (2019) assessed the RTK positioning accuracy of combined BDS-3/BDS-2, BDS-3 only, and BDS-2 only solutions using their stochastic model. The results demonstrated that the accuracy of RTK positioning was improved by $65 \%$ by comparing the BDS-2/BDS- 3 case with the BDS- 2 only case. Shi et al. (2020) further revealed that the additional BDS-3 observations accelerated the RTK positioning convergence for medium-long baseline compared with BDS-2 only solution. Zhang et al. (2020a) indicated that RTK positioning was feasible with the BDS-3 primary system. The achievable positioning accuracy for the involved short baseline was better than $1 \mathrm{~cm}$ for both BDS -3 only and combined BDS-2/BDS- 3 solutions.

Although some initial assessments of BDS-2/BDS-3 RTK were reported, only a few studies analyzed the BDS-3 only RTK positioning performance because the number of visible BDS-3 satellites was limited at that time with the BDS-3 primary system (Hou et al. 2019; Zhang et al. 2020a). The number of the visible satellites were four to six for only a relatively short observation period. With the completion of the BDS-3 full constellation, the situation has been changed. It is worthwhile to carry out a comprehensive performance evaluation of the RTK positioning with the BDS-3 full constellation and its combination with BDS-2 full constellation.

Furthermore, BDS-2 and BDS-3 are two generations of BDS operated and maintained by their individual control systems (Song et al. 2020). Due to various factors, e.g., different quality of time-keeping clocks and different receiver types in the ground monitoring network, systematic inter-system biases exist between BDS-3 and BDS-2, thereby affecting the achievable accuracy for combined BDS-3/BDS-2 single point positioning and precise point positioning. In such cases, BDS-2 and BDS-3 should be considered as two separate systems, and additional inter-system bias should be considered (Jiao et al. 2019; Song et al. 2020; Zhang et al. 2020b; Zhao et al. 2020). About combining BDS-3/BDS-2 for precise RTK positioning, previous studies either treated them directly as two separate constellations and used the classical loosely combined or intra-system model, i.e., one satellite was selected as the reference satellite for BDS-2 and BDS-3, separately, or did not explicitly present their approaches (Hou et al. 2019; Zhang et al. 2019b, c, 2020a; Shi et al. 2020). To fully utilize the interoperability between BDS-3 and BDS-2, the tightly combined doubledifferencing (i.e., a single reference satellite was selected for all BDS-3 and BDS-2 satellites) of observations from common frequencies (i.e., the legacy B1I/B3I) should be employed. The benefits of applying such a tightly combined model have been investigated intensively. The results demonstrated that the tightly combined model can improve RTK performance, particularly in challenging observational conditions where only a limited number of satellites and single-frequency observations were tracked (Odijk et al. 2017; Wu et al. 2017, 2019b). However, users must carefully consider the Differential Inter-System Biases (DISBs) when using this approach, even though the frequency of the involved observations from different systems is identical. Once the DISBs are carefully considered, the satellites from different Global Navigation Satellite Systems (GNSSs) can be used as if they were from a single GNSS constellation (Odijk and Teunissen 2013; Paziewski and Wielgosz 2015). Mi et al. (2020) preliminarily estimated the phase and code DISBs of BDS-3/BDS-2 B1I/B3I signals between two Trimble Alloy receivers and discovered that they were zero for the same receiver types, as expected. However, whether non-zero DISBs of BDS-3/BDS-2 B1I/B3I signals exist between different receiver types is yet to be elucidated, necessitating further investigations.

In this study, we evaluated the single-epoch short baseline RTK performance of the BDS-3 full constellation as well as its tight combination with the BDS-2 full constellation. We first investigated the characteristics of the phase and code DISBs between BDS-3/BDS-2 B1I/ B3I signals. Then we preliminarily evaluated the RTK positioning performance using single- and dual-frequency BDS-3/BDS-2 observations. Both the static and kinematic datasets collected in Wuhan were processed and analyzed to demonstrate the RTK performance of tightly combined BDS-3/BDS-2 and BDS-3 only solutions by ambiguity resolution and positioning accuracy. 
Furthermore, the benefits of tightly combined BDS-3/ BDS-2 solution with respect to BDS-3 only and BDS-2 only solutions were evaluated. It is noteworthy that although the navigation signals of the last BDS-3 GEO satellite were not tracked in our tests, it did not affect our performance assessment of the BDS-3 full constellation.

In this paper, we first present an overview of the current BDS-3/BDS-2 constellations. The existence and characteristics of phase and code DISBs between BDS-3/ BDS-2 B1I/B3I signals are investigated in "Feasibility of tightly combined BDS-2 and BDS-3 B1I/B3I RTK without additional DISBs" section. "Performance assessment of tightly combined BDS-2/BDS-3 RTK" presents the performance assessment of tightly combined BDS-3/ BDS-2 RTK using both single- and dual-frequency observations. Conclusions are given in "Conclusions and discussions" section.

\section{Current BDS-2/BDS-3 constellations}

BDS-2 has been in full operation with 14 satellites, comprising four MEO, five GEO, and five IGSO satellites since December 27, 2012. Another three BDS-2 replacement satellites (two GEO and one IGSO) were launched in 2016, 2018, and 2019 (CSNO-TARC 2020). Currently (early July 2020), BDS can provide open services with 44 operational satellites, including 15 BDS-2 and 29 BDS-3 satellites (see Table 2). As an example, Fig. 1 demonstrates the sky plot and availability of BDS-3 and BDS-2 satellites at the Multi-GNSS EXperiment (MGEX) station WUH2 in Wuhan on June 21, 2020. As shown, all operational BDS-3/BDS-2 satellites listed in Table 2 were observed. Note that BDS-3 GEO satellites C59 and C60 currently transmit only B1I/B3I signals, which means that only 27 among 29 BDS-3 operational satellites transmit new B1C/B2a/B2b signals, whereas they all transmit the legacy B1I/B3I signals. This could cause the number of visible BDS-3 satellites with B1I/B3I signals different from that with $\mathrm{B} 1 \mathrm{C} / \mathrm{B} 2 \mathrm{a}$ signals in the Asia-Pacific region. We will show this in the following analysis.

\section{Feasibility of tightly combined BDS-2 and BDS-3 B1I/B3I RTK without additional DISBs}

Previous studies have demonstrated that the values of code and phase DISBs depend on receiver types of the involved receivers at both endpoints of a baseline. If the involved receivers are of the same type and firmware version, the DISBs are about zero. Otherwise they are generally non-zero. Considering that they are generally stable in time even if the receivers restart, they can be accurately calibrated and corrected in advance (Odijk and Teunissen 2013; Yuan and Zhang 2014; Paziewski and Wielgosz 2015). Mi et al. (2020) estimated the DISBs of BDS-3/BDS-2 B1I/ B3I signals between two Trimble Alloy receivers. It was discovered that they were in fact absent for the same receiver types. In this section, we focus on estimating and analyzing the characteristics of code and phase DISBs between overlapping frequencies of BDS-3/BDS-2 B1I/B3I signals with both the same and different receiver types. The feasibility of tightly combined BDS-3/BDS-2 B1I/B3I RTK without additional DISBs will be investigated and identified.

\section{DISB estimation approach}

Assume that B1I/B3I observations from $n_{B}$ BDS-2 satellites and $n_{*}$ BDS- 3 satellites are observed simultaneously by two receivers at both endpoints of a zero or short baseline. These two receiver sites are denoted by base station $b$ and rover station $r$. If BDS- 2 and BDS-3 are considered as two separate systems and only a single BDS-2 satellite $\left(1_{B}\right)$ is selected as the reference satellite for all the BDS-2 and BDS-3 satellites, then the tightly combined double-differenced observation equation with DISB estimation for short baselines is expressed as (Wu et al. 2017, 2019b):

$$
\left\{\begin{array}{l}
\phi_{b r, f}^{1_{B} s_{B}}=\rho_{b r}^{1_{B} s_{B}}+\lambda_{f} N_{b r, f}^{1_{B} s_{B}}+\varepsilon_{b r, f}^{1_{B} s_{B}} \\
\phi_{b r, f}^{1_{B} s_{*}}=\rho_{b r}^{1_{B} s_{*}}+\lambda_{f}\left(N_{b r, f}^{1_{*} s_{*}}+\bar{\delta}_{b r, f}^{B *}\right)+\varepsilon_{b r, f}^{1_{B} s_{*}} \\
P_{b r, f}^{1_{B} s_{B}}=\rho_{b r}^{1_{B} s_{B}}+e_{b r, f}^{1_{B} s_{B}} \\
P_{b r, f}^{1_{B} s_{*}}=\rho_{b r}^{1_{B} s_{*}}+d_{b r, f}^{B *}+e_{b r, f}^{1_{B} s_{*}}
\end{array}\right.
$$

where $s_{B}$ and $s_{*}$ denote BDS-2 and BDS-3 satellites, respectively, and $s_{B}=2_{B}, \ldots, n_{B}, s_{*}=1_{*}, 2_{*}, \ldots, n_{*} ; f$ is

Table 2 Status of operational BDS-2 and BDS-3 satellites as of July 2020

\begin{tabular}{llll}
\hline System & Orbit & Number & PRN \\
\hline BDS-2 & GEO & 5 & C01-C05 \\
& IGSO & 7 & $C 06-C 10, C 13, C 16$ \\
& MEO & 3 & $C 11, C 12, C 14$ \\
BDS-3 & GEO & 2 & $C 59, C 60$ \\
& IGSO & 3 & $C 38, C 39, C 40$ \\
MEO & 24 & $C 19-C 30, C 32-C 37, C 41-C 46$ \\
\hline
\end{tabular}




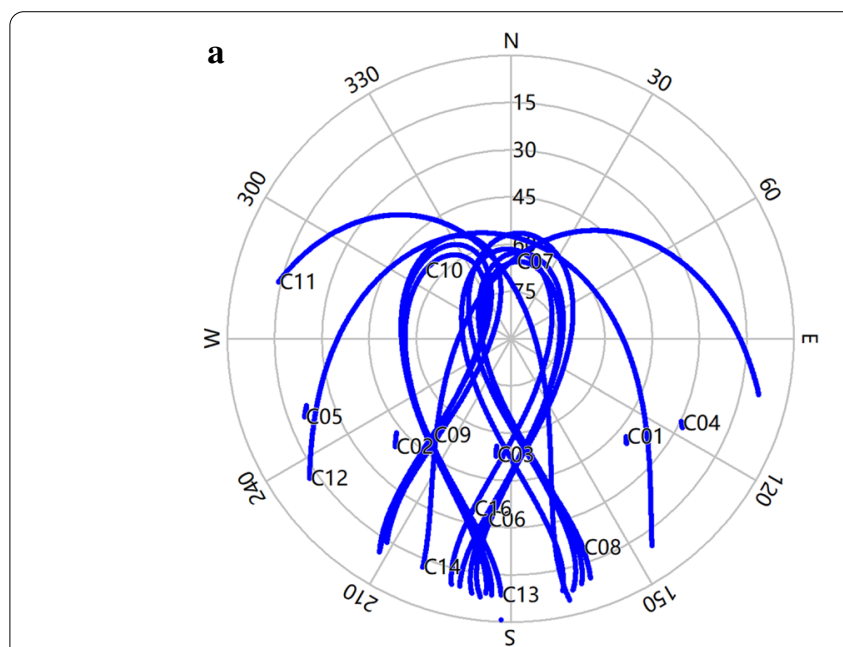

BDS-2

c

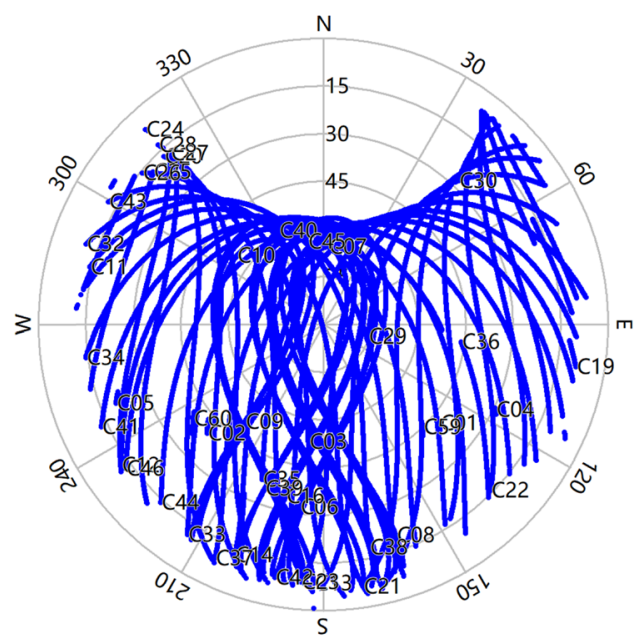

BDS-2/BDS-3

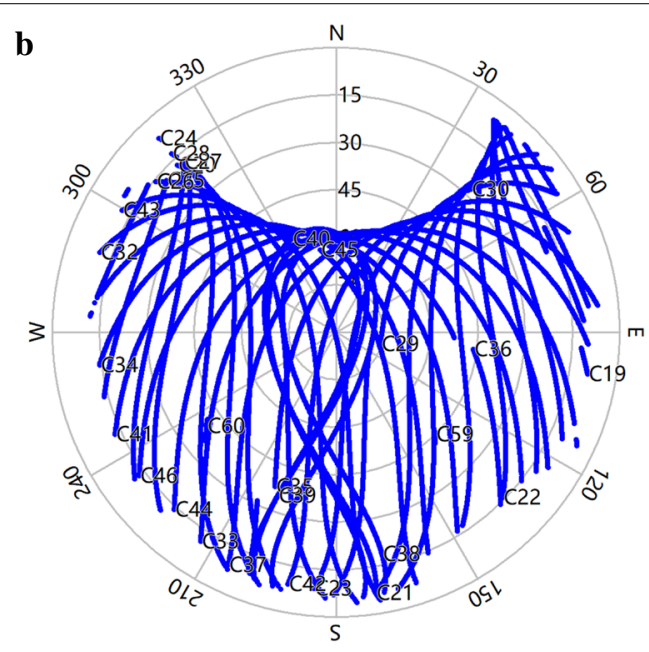

BDS-3

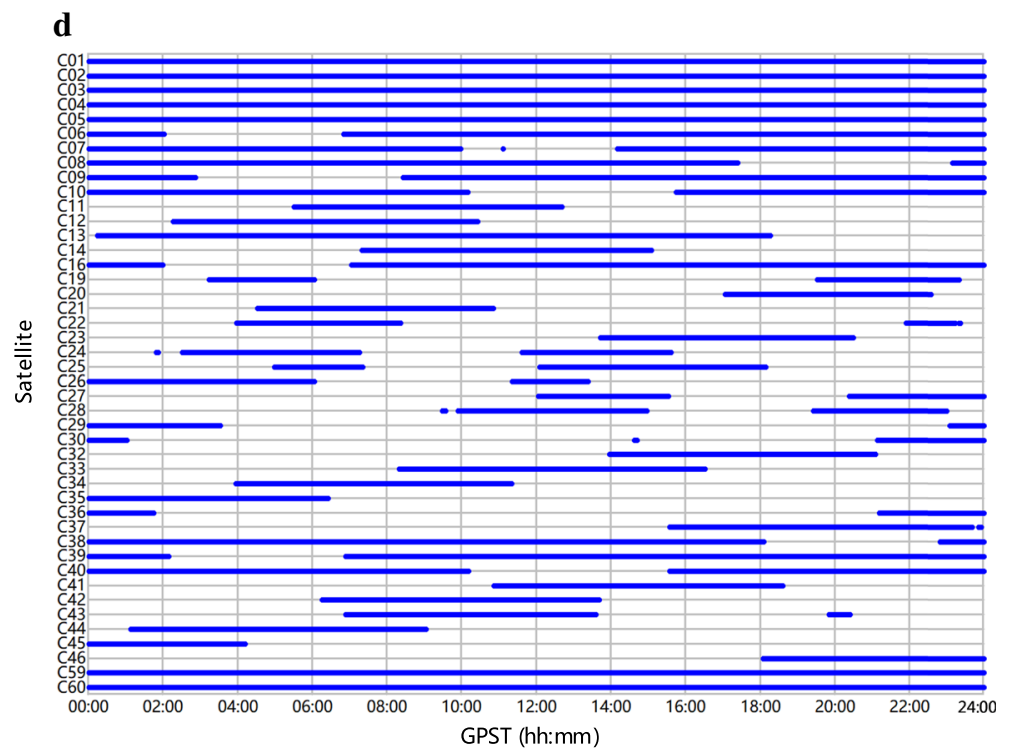

Fig. 1 Sky plot and availability of BDS-3 and BDS-2 satellites at MGEX station WUH2 in Wuhan on June 21, 2020

the frequency of the B1I/B3I observations; $P$ and $\phi$ illustrate the code and phase observations in meters; $\rho$ denotes the geometric distance between the receiver and satellite; $\lambda$ represents the wavelength, while $N$ is the integer ambiguity in cycles; $e$ and $\varepsilon$ denote the observation noise in code and phase, respectively; $\bar{\delta}_{b r, f}^{B *}=\delta_{b r, f}^{B *}+N_{b r, f}^{1_{B} 1_{*}}$ is the reorganized phase DISB parameter that are formed by combining the double-differenced ambiguity between reference satellites of BDS-2 and BDS-3 $\left(N_{b r, f}^{1_{1} 1_{*}}\right)$ with the original phase DISB parame$\operatorname{ter}\left(\delta_{b r, f}^{B *}\right) ; d_{b r, f}^{B *}$ is the code DISB parameter.

\section{Data collection}

The BDS-3/BDS-2 data collected from the static experiments at the Wuhan University campus were used. In the static experiments, a pair of geodetic iGMAS receivers (Zhang et al. 2017), a pair of Trimble Alloy receivers (firmware version 6.05), and two Trimble Zephyr Geodetic 2 antennas were employed. The antennas were located on the roof of our department (School of Geodesy and Geomatics (SGG), Wuhan University). The configuration of the receivers and antennas at the SGG stations is shown in Fig. 2. Meanwhile, raw BDS-2/BDS-3 data collected at the MGEX station WUH2 (equipped with Javad TRE_3 receiver (firmware 


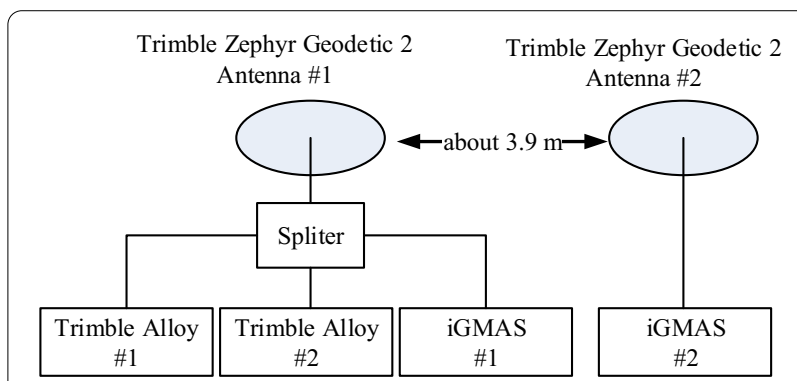

Fig. 2 Configuration of multi-GNSS receivers and antennas at SGG stations

version 3.7.9) and JAVRINGANT_G5T NONE antenna) were also used. The station WUH2 was also located in Wuhan University and separated by approximately $371 \mathrm{~m}$ from the SGG building. The locations of the stations and the observational conditions of the SGG stations are shown in Fig. 3. The employed GNSS receivers and the tracking modes of the BDS signals are listed in Table 3.

\section{DISB estimation results}

Using Eq. (1), the phase and code DISBs for the B1I/ B3I signals were epoch-wise estimated by fixing the precisely known receiver coordinates. The datasets were collected in the static experiments at the Wuhan University campus. The data for the zero and short baselines were collected under an elevation cut-off angle of $10^{\circ}$ on June 9, 2020. Figure 4 shows the estimated phase and code DISBs between two Trimble Alloy receivers for BDS-3/BDS-2 B1I/B3I signals. Figure 5 shows the estimated phase and code DISBs between the Trimble Alloy and Javad TRE_3 receivers. Table 4 gives the estimated phase and code DISBs [the average (AVE) values together with the standard deviations (STDs)] for both the same and different receiver types. As shown in Figs. 4, 5, and Table 4, regardless of the same or different receiver types used at both endpoints of a baseline, the estimated phase and code DISBs were all approximately zero and thus negligible. For example, the phase and code DISBs for two Trimble Alloy receivers were $0 / 0$ cycles and $-0.08 / 0.01 \mathrm{~m}$ for B1I/B3I signals, respectively, and for the Trimble Alloy and Javad TRE_3 receivers, the values were $-0.008 /-0.001$ cycles and $-0.28 / 0.25 \mathrm{~m}$, respectively. These results reveal that BDS- 3 and BDS- 2 can be considered as one single constellation without additional DISBs when B1I/B3I observations are used for precise relative positioning, implying full interoperability between BDS-2 and BDS-3.
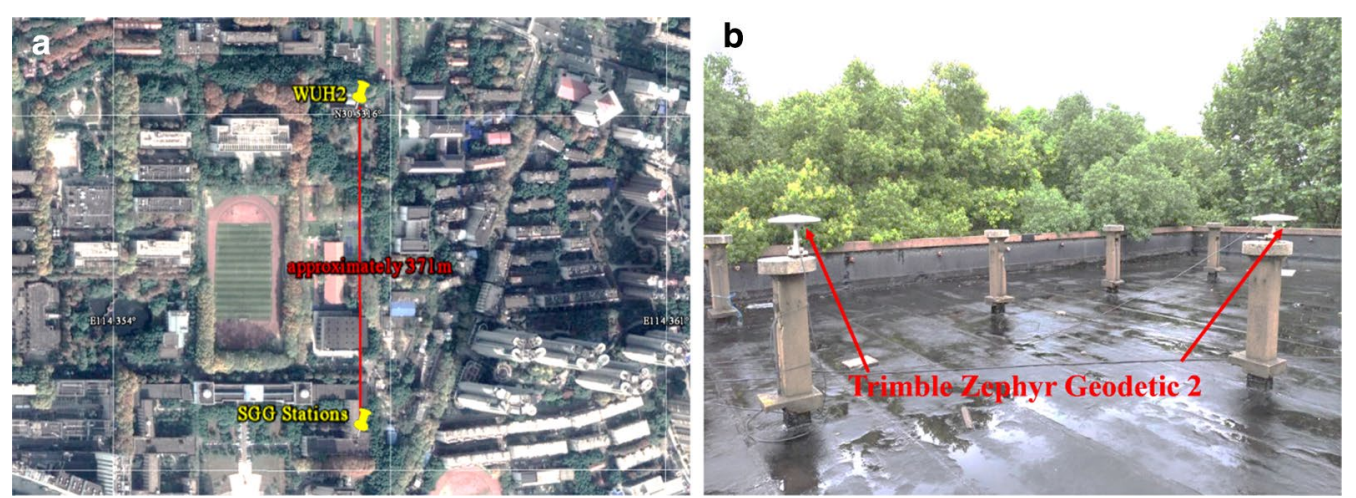

Fig. 3 Locations of the stations and observational condition of SGG stations

Table 3 Employed GNSS receivers and BDS observables with tracking modes that supported in RINEX version 3.04 (IGS and RTCM-SC104 2018)

\begin{tabular}{|c|c|c|c|c|c|c|c|c|}
\hline Receiver type & $\begin{array}{l}\text { Firmware } \\
\text { version }\end{array}$ & $\mathrm{B} 1 \mathrm{C}$ & B2a & $\mathrm{B} 2 \mathrm{~b}$ & B1I & B3I & B2I & Note \\
\hline iGMAS & 1.1 .2 & L1D & L5D & L7D & L2I & L6। & L7I & Could not track C59, C60 \\
\hline Trimble Alloy & 6.05 & L1X & L5X & L7D & $\mathrm{L} 2 \mathrm{I}$ & $\mathrm{L} 6 \mid$ & L7I & $\begin{array}{c}\text { Tracked all BDS-3/BDS-2 } \\
\text { operational satellites }\end{array}$ \\
\hline Javad TRE_3 & 3.7 .9 & $\mathrm{~L} 1 \mathrm{X}$ & L5X & $\mathrm{L} 7 \mathrm{Z}$ & $\mathrm{L} 2 \mathrm{I}$ & L6I & L7I & $\begin{array}{c}\text { Tracked all BDS-3/BDS-2 } \\
\text { operational satellites }\end{array}$ \\
\hline
\end{tabular}



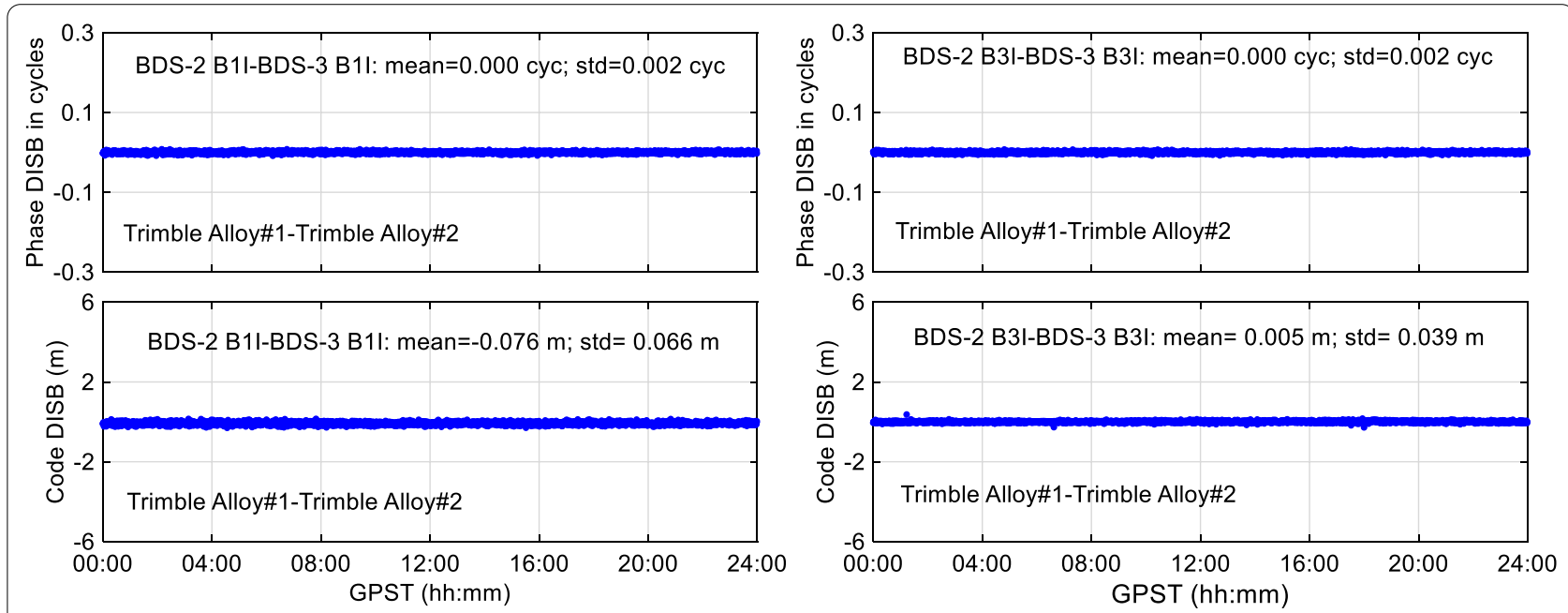

Fig. 4 Estimated phase and code DISBs between two Trimble Alloy receivers
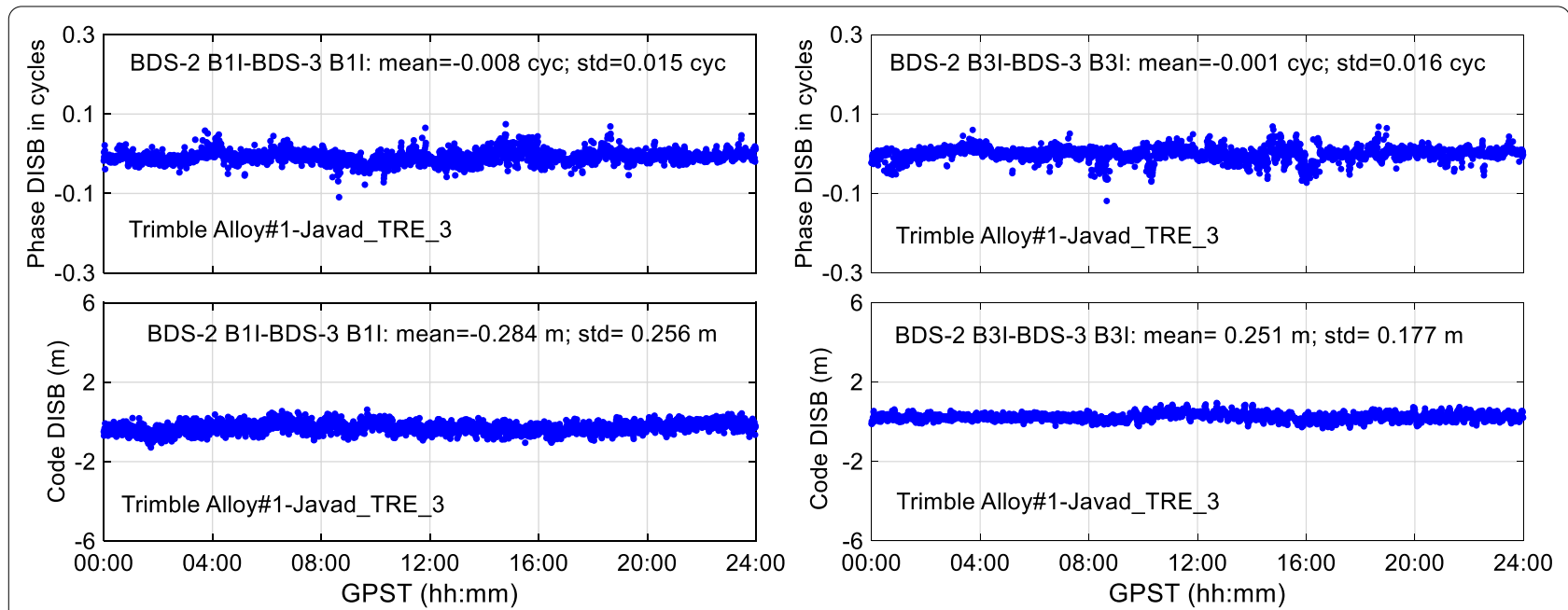

Fig. 5 Estimated phase and code DISBs between Trimble Alloy and Javad TRE_3 receivers

Table 4 Phase and code DISB estimates for both the same and different receiver types

\begin{tabular}{|c|c|c|c|c|c|c|c|c|c|}
\hline \multirow[t]{3}{*}{ Receiver types } & \multirow{3}{*}{$\begin{array}{l}\text { Baseline } \\
\text { distance }\end{array}$} & \multicolumn{4}{|c|}{ Phase DISB in cycles } & \multicolumn{4}{|c|}{ Code DISB (m) } \\
\hline & & \multicolumn{2}{|l|}{ B1I } & \multicolumn{2}{|l|}{ B3I } & \multicolumn{2}{|l|}{ B1I } & \multicolumn{2}{|l|}{ B3I } \\
\hline & & AVE & STD & AVE & STD & AVE & STD & AVE & STD \\
\hline $\begin{array}{l}\text { Trimble Alloy\#1 } \\
\text { Trimble Alloy\#2 }\end{array}$ & Zero & 0.000 & 0.002 & 0.000 & 0.002 & -0.08 & 0.07 & 0.01 & 0.04 \\
\hline $\begin{array}{l}\text { Trimble Alloy\#1 } \\
\text { Javad TRE_3 }\end{array}$ & Short & -0.008 & 0.015 & -0.001 & 0.016 & -0.28 & 0.26 & 0.25 & 0.18 \\
\hline $\begin{array}{l}\text { Trimble Alloy\#1 } \\
\text { iGMAS \#1 }\end{array}$ & Zero & -0.001 & 0.003 & -0.001 & 0.004 & -0.03 & 0.20 & 0.29 & 0.09 \\
\hline $\begin{array}{l}\text { iGMAS\#1 } \\
\text { iGMAS \#2 }\end{array}$ & Short & 0.002 & 0.009 & 0.003 & 0.014 & -0.03 & 0.39 & 0.11 & 0.27 \\
\hline $\begin{array}{l}\text { Javad TRE_3 } \\
\text { iGMAS\#1 }\end{array}$ & Short & 0.009 & 0.014 & -0.003 & 0.014 & 0.23 & 0.30 & 0.03 & 0.16 \\
\hline
\end{tabular}




\section{Performance assessment of tightly combined BDS-2/BDS-3 RTK}

In this part, we carry out performance evaluation of single-epoch short-baseline RTK positioning with current BDS-2 and BDS-3 full constellations. The tightly combined BDS-3/BDS-2 solution is then compared with BDS -3 only and BDS- 2 only solutions with observations from two common signals (B1I and B3I). For the BDS-3 only solutions, we also compared the RTK performance of new B1C/B2a navigation signals with that of the legacy B1I/B3I signals. Four processing schemes (see Table 5) were adopted for both the single- and dual-frequency solutions. As mentioned above, BDS- 2 and BDS-3 can be regarded as one constellation if B1I/B3I observations are used in precise relative positioning, therefore only one single-reference satellite was selected for all BDS-2 and BDS-3 satellites in the schemes "BDS-2/BDS-3 B1I" and "BDS-2/BDS-3 B1I/B3I".

All the data were processed epoch-by-epoch using the double-differenced model with software KinPOS v3.0, developed by Wuhan University. Because our study was on short baselines, the double-differenced ionospheric and tropospheric delays were negligible. Thus, only the Three-Dimensional (3D) baseline vector and the doubledifferenced ambiguities were estimated in each epoch. The estimated ambiguities from float solution were then fixed to integers based on the method of Least-squares AMBiguity Decorrelation Adjustment (LAMBDA) (Teunissen 1995), among which the popular ratio test with a threshold of 2.0 was adopted for ambiguity validation. Additionally, different elevation cut-off angles $\left(10^{\circ}\right.$, $15^{\circ}, 20^{\circ}, 25^{\circ}, 30^{\circ}, 35^{\circ}$, and $40^{\circ}$ ) were set to simulate different observational conditions and satellite visibilities.

Meanwhile, the following elevation-dependent weighting function (Herring et al. 2018) was used in the stochastic model:

$$
\sigma^{2}(\theta)=a^{2}+\frac{b^{2}}{\sin ^{2}(\theta)}
$$

where $\sigma^{2}(\theta)$ denotes the variance of undifferenced observation, $\theta$ represents the satellite elevation angle. $a$ and $b$ are model coefficients with specified empirical values. Here, both $a$ and $b$ are set to $0.003 \mathrm{~m}$ for phase observations of BDS-3 B1I/B1C/B2a/B3I and BDS-2 B1I/B3I, while $0.3 \mathrm{~m}$ for code observations.

\section{Evaluation statistics}

The performance of BDS-3/BDS-2 RTK positioning was evaluated by ambiguity resolution success rate, Ambiguity Dilution of Precision (ADOP), together with positioning accuracy.

ADOP is a well-known theoretical figure of merit for inferring the average precision of estimated ambiguities from float solution, which is expressed as (Teunissen 1997):

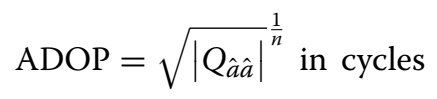

where $n$ denotes the number of float ambiguities; $\left|Q_{\hat{a} \hat{a}}\right|$ illustrates the determinant of variance-covariance matrix for the estimated ambiguities from float solution. Smaller ADOP value implies higher average precision of the estimated float ambiguities, while an ADOP value smaller than 0.12 cycles indicates that the achievable ambiguity resolution success rate are theoretically higher than 99.9\% (Odijk and Teunissen 2008).

In addition to the theoretical analysis of the ADOP, we used the success rate to demonstrate the empirical performance of ambiguity resolution, which is defined as the number of epochs with ambiguities correctly resolved divided by the total epoch numbers, thereby reflects the availability of reliable and accurate RTK positioning. The ambiguities are regarded as correctly resolved only if the test ratio is no less than a specified threshold (2.0 in this study). Meanwhile, the positioning errors should be less than $5 \mathrm{~cm} / 5 \mathrm{~cm} / 10 \mathrm{~cm}$ in the East (E)/North $(\mathrm{N}) / \mathrm{Up}(\mathrm{U})$ components compared with "true" baseline vector, which is the post-processed baseline fixed solution with BDS-3/ BDS-2 observations over the entire observation period. Root-Mean-Square (RMS) of the positioning error series from those correctly resolved solutions was computed and compared to evaluate the RTK positioning accuracy as well.

\section{Experimental results}

In this research, the data for both the static and kinematic modes collected in Wuhan were analyzed. The static data were collected in the Wuhan University campus, whereas the kinematic data were collected by a car along Liangzi Lake Avenue.

Table 5 Processing schemes

\begin{tabular}{lllll}
\hline Observations & Scheme 1 & Scheme 2 & Scheme 3 & Scheme 4 \\
\hline Single-frequency & BDS-2 B11 & BDS-3 B11 & BDS-3 B1C & BDS-2/BDS3 B11 \\
Dual-frequency & BDS-2 B11/B31 & BDS-3 B11/B31 & BDS-3 B1C/B2a & BDS-2/BDS3 B11/B31 \\
\hline
\end{tabular}




\section{Static test}

The static data were also collected from the static experiments performed in the Wuhan University campus (as demonstrated in the "Data collection"), on June 21, 2020. A short baseline with a length of approximately $371 \mathrm{~m}$ was selected, whose endpoints were a Trimble Alloy \#2 receiver at a SGG station and a Javad TRE_3 receiver at WUH2. The BDS-2/BDS-3 satellites were observed with sampling interval of $30 \mathrm{~s}$ and elevation cut-off angle of $10^{\circ}$.

Figure 6 reveals the number of visible BDS-2 and BDS-3 satellites at the $10^{\circ}$ elevation cut-off angle as well as their Position Dilution of Precision (PDOP) series. It was observed that the numbers of tracked satellites were 8-14 for BDS-2 and 8-13 for BDS-3. For combined BDS-3/BDS-2, the number of tracked satellites increased to $17-24$, nearly double that of each system. The PDOP values were approximately $1.8-4.5,1.4-3.8$, and $1.0-2.5$ for BDS-2, BDS-3, and combined BDS-3/BDS-2, respectively. The average number of visible satellites for BDS-2, BDS-3, and combined BDS-3/BDS-2 during the day were $10.7,10.2$, and 20.9, while the average PDOPs were 2.39,
2.00 , and 1.42, respectively. These results demonstrate that the geometry strength was improved significantly by combining BDS-3/BDS-2. Meanwhile, because enough BDS-3 satellites with good geometry were available, a relatively superior RTK performance was expected for BDS3 . It is noteworthy that the frequent jumps in the number of observed satellites and PDOP series for BDS-2 were primarily caused by the intermittent tracking of $\mathrm{C} 05$.

Figure 7 shows the average number of BDS-3/BDS-2 satellites and their PDOP values during the entire observation period with elevation cut-off angles of $10^{\circ}, 15^{\circ}$, $20^{\circ}, 25^{\circ}, 30^{\circ}, 35^{\circ}$, and $40^{\circ}$. Considering that BDS-3 GEO satellites $\mathrm{C} 59$ and C60 transmit only B1I/B3I signals currently but not $\mathrm{B} 1 \mathrm{C} / \mathrm{B} 2 \mathrm{a}$ signals, we distinguished the visible satellites of B1I/B3I signals from that of B1C/B2a signals. Focusing on visible satellites with B1I/B3I signals, we observe that the average visible BDS-2 and BDS-3 satellites decreased from approximately 10.7 and 10.2 with the elevation cut-off angle of $10^{\circ}$ to 7.4 and 6.1 with the elevation cut-off angle of $40^{\circ}$, and their corresponding PDOP values increased from approximately 2.39 and 2.00 to 5.65 and 7.03 , respectively. For the combined
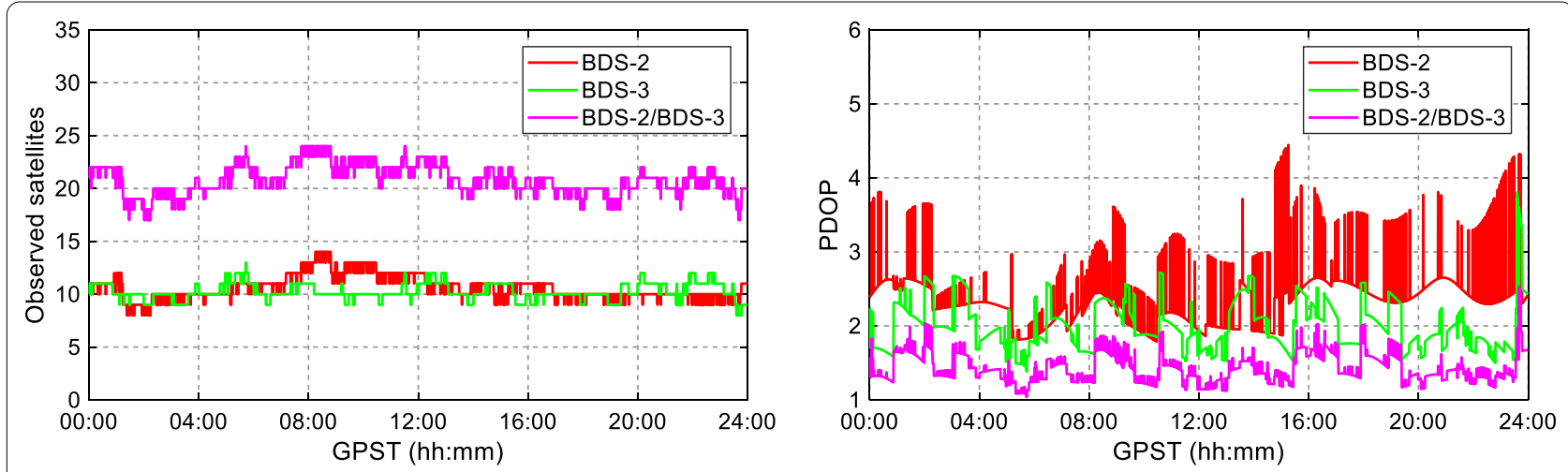

Fig. 6 Observed BDS-2 and BDS-3 satellites and their PODP values with an elevation cut-off angle of $10^{\circ}$
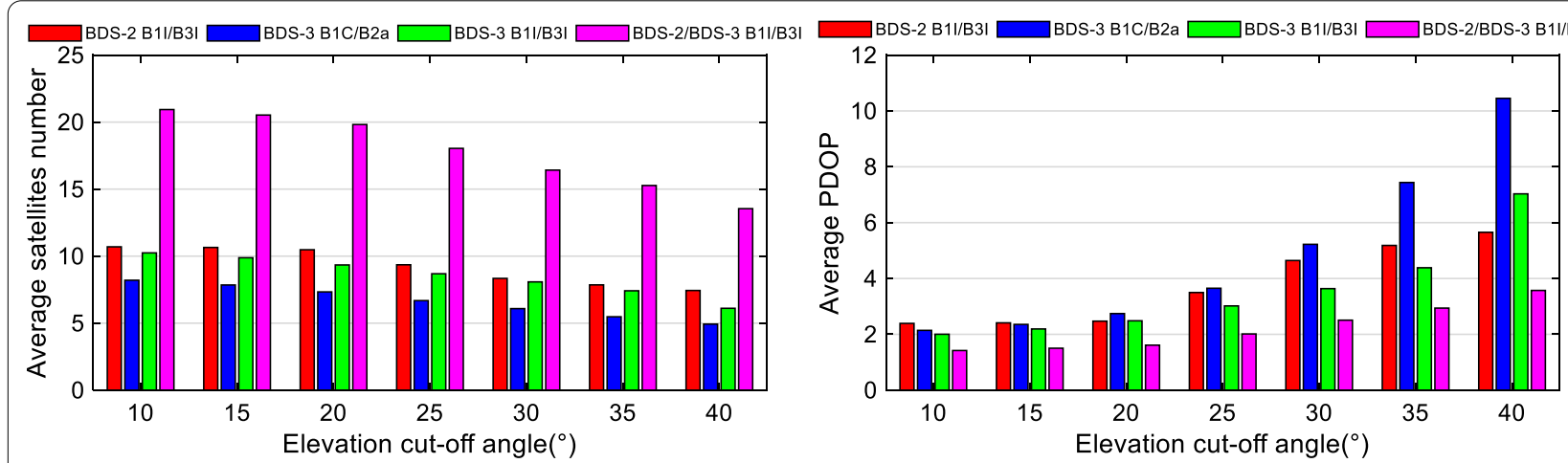

Fig. 7 Average number of observed BDS-3/BDS-2 satellites and their PDOP values during the observation period under different elevation cut-off angles 

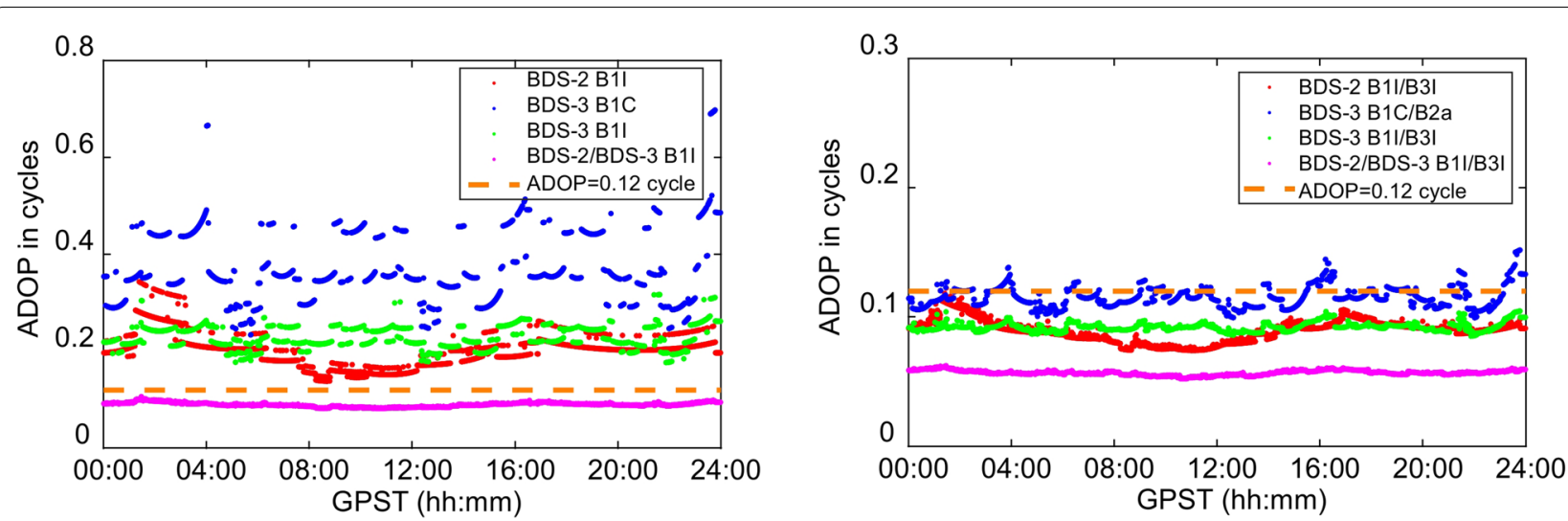

Fig. 8 ADOP from single-frequency (left) and dual-frequency (right) observations at an elevation cut-off angle of $10^{\circ}$ for BDS-2 only, BDS-3 only, and tightly combined BDS-3/BDS-2 solutions

BDS-3/BDS-2, the visible satellite number reached up to approximately 13.5 (with a PDOP value of approximately 3.57 ) even with the elevation cut-off angle of $40^{\circ}$, indicating its relatively good geometry and its potential in providing reliable RTK positioning in challenging and harsh observational conditions. Moreover, we observed that although more BDS-2 satellites were observed than BDS-3 under all elevation cut-off angles, larger PDOP values were obtained for BDS-2 except for the elevation cut-off angle of $40^{\circ}$ case, indicating a better geometry of BDS-3 with respect to that of BDS-2 under the current constellations. This is reasonable considering that the current BDS-2 constellation comprises three MEO, five GEO, and seven IGSO operational satellites, while BDS-3 constellation comprises $24 \mathrm{MEO}$, two GEO, and three IGSO operational satellites. Regarding BDS-3, it was discovered that its PDOP values for B1I/B3I signals were smaller than that for B1C/B2a signals, particularly under high elevation cut-off angles. These results confirmed that the BDS-3 GEO satellites contributed to a better geometry in the Asia-Pacific region.

Figure 8 shows the epoch-by-epoch ADOP series using single- and dual-frequency observations under the elevation cut-off angle of $10^{\circ}$ for BDS-2 only, BDS-3 only, and tightly combined BDS-3/BDS-2 solutions, respectively. For B1I/B3I signals, we discovered that the single-frequency ADOP values of BDS-3 only and BDS- 2 only solutions were larger than 0.12 cycles, whereas they were smaller than 0.12 cycles for tightly combined BDS-3/BDS-2 solution. The average ADOP values were $0.200,0.229$, and 0.090 cycles for the BDS-2 only, BDS-3 only, and tightly combined BDS-3/BDS-2 solutions, respectively. Theoretically, these results imply that a reliable single-epoch single-frequency ambiguity resolution was infeasible for BDS-2 only and BDS-3 only solutions, but feasible for tightly combined BDS-3/
BDS-2 solution. With respect to dual-frequency solutions, the ADOP series were generally below 0.12 cycles for BDS-2 only, BDS-3 only, as well as tightly combined BDS-3/BDS-2 solutions, and the average ADOP values were $0.089,0.092$, and 0.057 cycles, respectively, indicating the feasibility of reliable single-epoch ambiguity resolution for all three dual-frequency solutions. Additionally, comparing the BDS-3 only solution using the new navigation B1C/B2a signals with that using the legacy B1I/B3I signals, it was discovered that the ADOP values for the BDS-3 $\mathrm{B} 1 \mathrm{C}$ and $\mathrm{B} 1 \mathrm{C} / \mathrm{B} 2 \mathrm{a}$ solutions were obviously larger than those for the BDS-3 B1I and B1I/ B3I solutions, which is reasonable considering that BDS-3 GEO satellites C59 and C60 transmit only B1I/ B3I navigation signals currently.

Figure 9 shows the ambiguity resolution success rates of single-frequency observations with different elevation cut-off angles. For B1I signal, it was demonstrated that the success rates for BDS-2 were slightly higher than those for BDS-3 at all elevation cut-off angles except for $30^{\circ}$. If elevation cut-off angle was below $25^{\circ}, 87-88 \%$ and $84-85 \%$ success rates were then obtained for BDS- 2 only and BDS-3 only solutions, respectively. With increasing elevation cut-off angle from $25^{\circ}$ to $40^{\circ}$, the success rates decreased dramatically from approximately $77.9-38.3 \%$ and $77.6-17.3 \%$ for BDS-2 only and BDS-3 only solutions, respectively. For tightly combined BDS-3/BDS-2 solutions, however, $100 \%$ success rate was obtained for the elevation cut-off angle below $40^{\circ}$, and $99.6 \%$ success rate for the elevation cut-off angle of $40^{\circ}$. These promising results show the potential of the tightly combined BDS-3/BDS-2 RTK in challenging and harsh observational conditions in the Asia-Pacific region. Consistent with previous ADOP results, the success rates were much lower for the BDS-3 B1C solutions than those for the BDS-3 B1I solutions. 


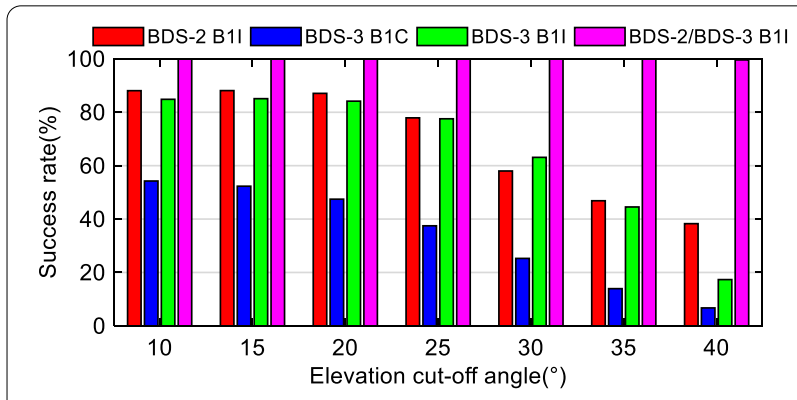

Fig. 9 Single-epoch ambiguity resolution success rates based on single-frequency observations

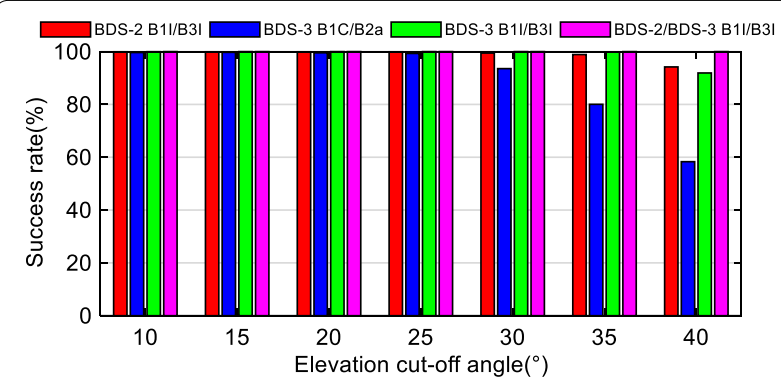

Fig. 10 Single-epoch ambiguity resolution success rates based on dual-frequency observations

Figure 10 shows the ambiguity resolution success rates based on dual-frequency observations at different elevation cut-off angles. It was observed that if the elevation cut-off angle was below $25^{\circ}$, almost $100 \%$ success rates were obtained for all the solutions. With increasing elevation cut-off angle from $25^{\circ}$ to $40^{\circ}$, the success rates of BDS-2 and BDS-3 B1I/B3I solutions decreased slightly to approximately $94.2 \%$ and $91.9 \%$, whereas those of the BDS-3 B1C/B2a solutions decreased dramatically to approximately $58.3 \%$. For the tightly combined BDS-3/ BDS-2 solutions, $100 \%$ success rates were achieved under all elevation cut-off angles. Hence, we conclude that tightly combined BDS-3/BDS-2 could significantly improve ambiguity resolution performance compared with BDS-2 or BDS-3 alone, particularly under challenging or severe observational conditions.

Figure 11 shows positioning errors in the $E, N$, and $U$ components for BDS-2, BDS-3, and tightly combined BDS-3/BDS-2 using dual-frequency observations at the elevation cut-off angle of $10^{\circ}$. The corresponding positioning accuracy (only for ambiguity fixed solutions) is listed in Table 6. As shown, the positioning errors were primarily within the range of -1.0 to $1.0 \mathrm{~cm}$ in the East and North components and the range of -4.0 to $4.0 \mathrm{~cm}$ in the Up component. About the B1I/B3I signals, the positioning accuracy for BDS-3 only solution (RMS values of $0.31 \mathrm{~cm} / 0.34 \mathrm{~cm} / 1.35 \mathrm{~cm}$ in the

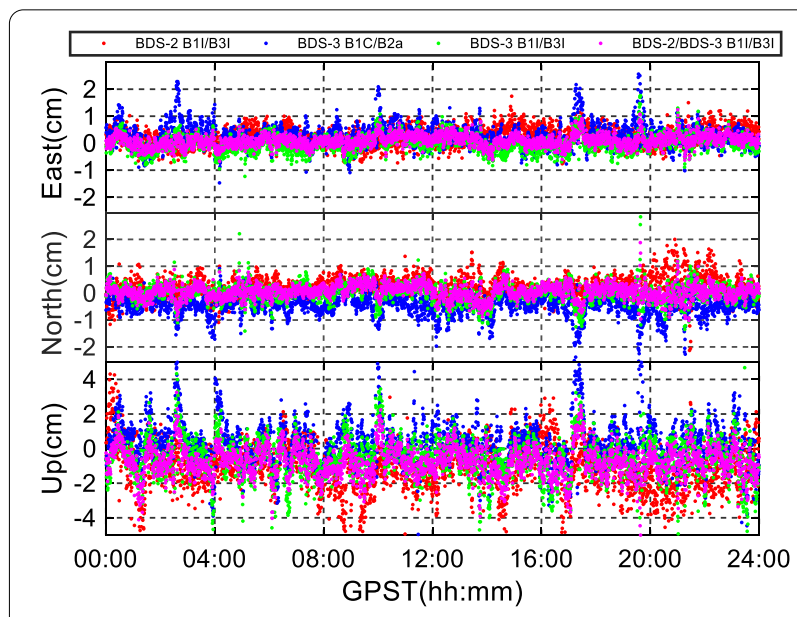

Fig. 11 The positioning errors with dual-frequency BDS-2/BDS-3 observations at the elevation cut-off angle of $10^{\circ}$

$E / N / U)$ was better than that for BDS-2 only solution $(0.41 \mathrm{~cm} / 0.44 \mathrm{~cm} / 1.74 \mathrm{~cm})$, showing good agreement with the previous PDOP results. If BDS-3 and BDS-2 are tightly combined, the positioning accuracy can then be significantly improved, i.e., the RMS values of $0.24 \mathrm{~cm} / 0.27 \mathrm{~cm} / 1.18 \mathrm{~cm}$ in the $E / N / U$. The $3 \mathrm{D}$ position RMS was $1.84 \mathrm{~cm}$ for BDS-2, $1.43 \mathrm{~cm}$ for BDS-3, and $1.23 \mathrm{~cm}$ for tightly combined BDS-3/BDS-2. The positioning accuracy for the BDS-3 B1C/B2a solution was slightly lower than the BDS-3 B1I/B3I solution. This is reasonable considering its higher PDOP values due to the absence of C59 and C60.

\section{Kinematic test}

A kinematic test was performed on June 24, 2020 from 06:45 to 08:00 GPS Time (GPST) in Wuhan, China. In the experiment, two Trimble Alloy receivers were used as the base and rover. The base receiver and antenna (Trimble Zephyr Geodetic 2) were located beside the Liangzi Lake Avenue, with an open-sky view. The rover receiver and antenna (Trimble Zephyr Model 2) were installed on top of a car driving along the Liangzi Lake Avenue, with an approximate speed of $50 \mathrm{~km} / \mathrm{h}$. Distance between rover and the base was approximately $0.3-7.9 \mathrm{~km}$ during the

Table 6 RMS of the single-epoch BDS-2/BDS-3 RTK positioning errors

\begin{tabular}{lllll}
\hline Observations & $\boldsymbol{E}(\mathbf{c m})$ & $\boldsymbol{N}(\mathbf{c m})$ & $\boldsymbol{U}(\mathbf{c m})$ & 3D $(\mathbf{c m})$ \\
\hline BDS-2 B11/B31 & 0.41 & 0.44 & 1.74 & 1.84 \\
BDS-3 B11/B31 & 0.31 & 0.34 & 1.35 & 1.43 \\
BDS-3 B1C/B2a & 0.52 & 0.62 & 1.36 & 1.58 \\
BDS-2/BDS-3 B11/B31 & 0.24 & 0.27 & 1.18 & 1.23 \\
\hline
\end{tabular}



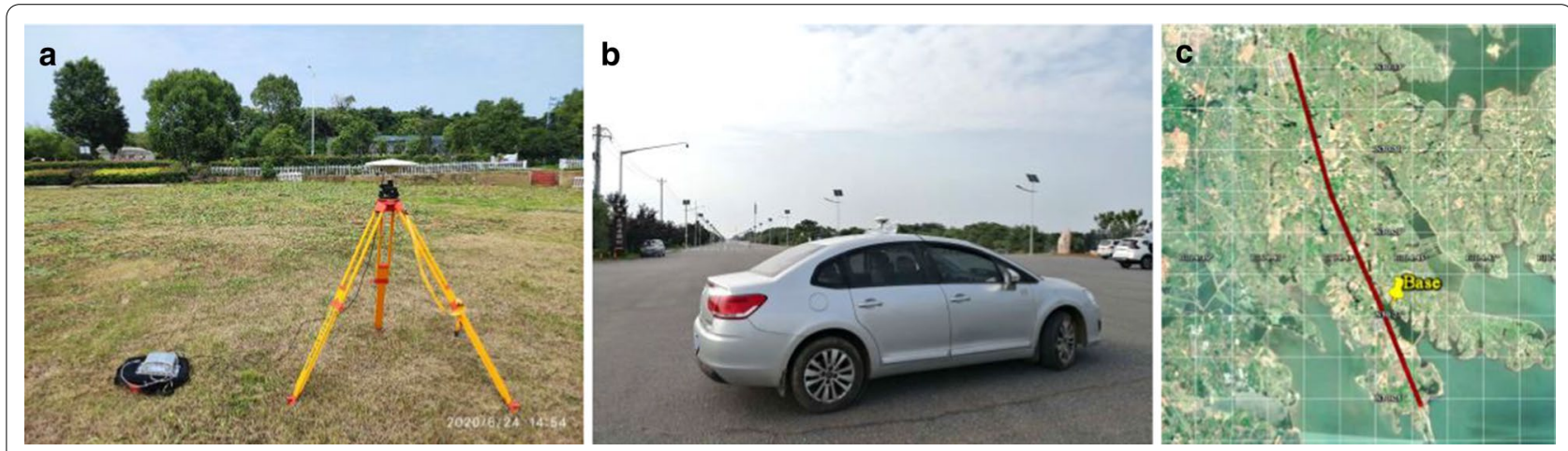

Fig. 12 Observational conditions of the base and rover station, and the trajectory of the car

entire observation period, and the data were collected at a sampling interval of one second with elevation cut-off angle of $10^{\circ}$. Figure 12 illustrates the observational condition of base and the rover station, as well as trajectory of the car. Because there were mainly some small trees along the both sides of Liangzi Lake Avenue, the observational condition of this kinematic test was relatively good without obvious obstructions.

Figure 13 gives the average number of observed BDS-3/ BDS-2 satellites and PDOP values during the observation period at the elevation cut-off angle of $10^{\circ}$. Unlike static test, the number of observed BDS-2 satellites were smaller than that of BDS-3. Specifically, the number of observed BDS-2, BDS-3, and combined BDS-3/ BDS-2 satellites during kinematic test were 7-11, 8-13, and $17-24$, respectively. The PDOP values were approximately $2.0-3.3,1.5-4.5$, and $1.2-1.9$, respectively. The average number of tracked BDS-2, BDS-3, and combined BDS-3/BDS-2 satellites was 10.4, 11.1, and 21.5, while the average PDOP was $2.37,1.84$, and 1.37 , respectively. It is noteworthy that the frequent jumps in the number of the observed satellites and the PDOP series for BDS-2 were primarily caused by the intermittent tracking of
C05, whereas the jumps of BDS-3 at approximately 07:00 GPST were primarily caused by intermittent tracking of C19, C36, and C45.

Figure 14 depicts the average number of observed BDS-3/BDS-2 satellites and the PDOP values during the test under different elevation cut-off angles. For the B1I/ B3I signals, the average number of the observed BDS-2, BDS-3, and combined BDS-3/BDS-2 satellites decreased from approximately 10.4, 11.1, and 21.5 under the elevation cut-off angle of $10^{\circ}$ to $6.2,7.3$, and 13.5 under the elevation cut-off angle of $40^{\circ}$, respectively, and their PDOP values increased from approximately $2.37,1.84$, and 1.37 to $6.77,4.92$, and 3.76 , respectively. The PDOP values of BDS-2 were obviously larger than those of BDS-3 except for the elevation cut-off angle of $20^{\circ}$, and a combination of BDS-3/BDS-2 provided a relatively good geometry even under an elevation cut-off angle of $40^{\circ}$.

Figure 15 shows the epoch-by-epoch ADOP series based on single- and dual-frequency observations at the elevation cut-off angle of $10^{\circ}$ for BDS-2, BDS-3, and tightly combined BDS-3/BDS-2 solutions, respectively. Similar as static test, the single-frequency ADOP values of the BDS-3 only and BDS- 2 only solutions were
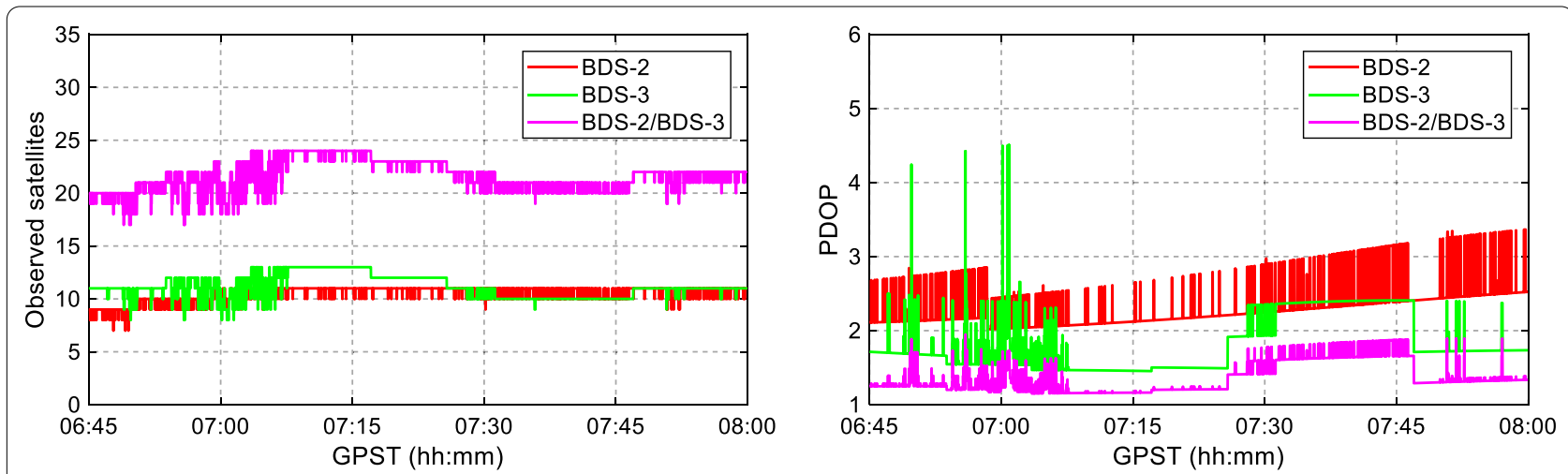

Fig. 13 Observed BDS-2 and BDS-3 satellites and PODP values with an elevation cut-off angle of $10^{\circ}$ 

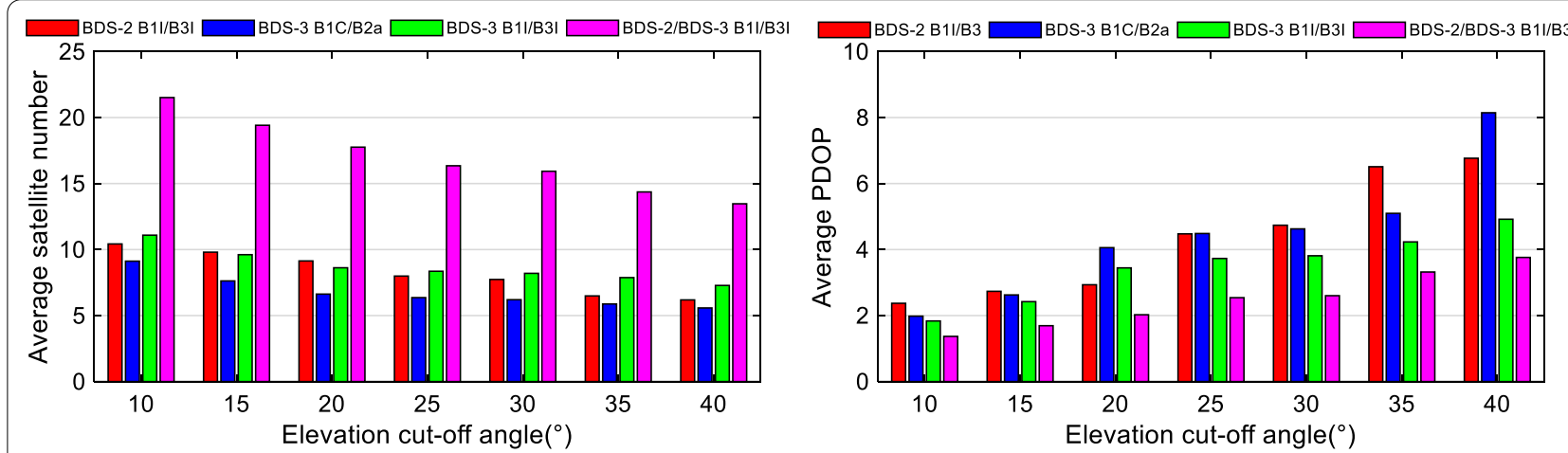

Fig. 14 The average number of observed BDS-3/BDS-2 satellites and their PDOP values during the observation period under different elevation cut-off angles
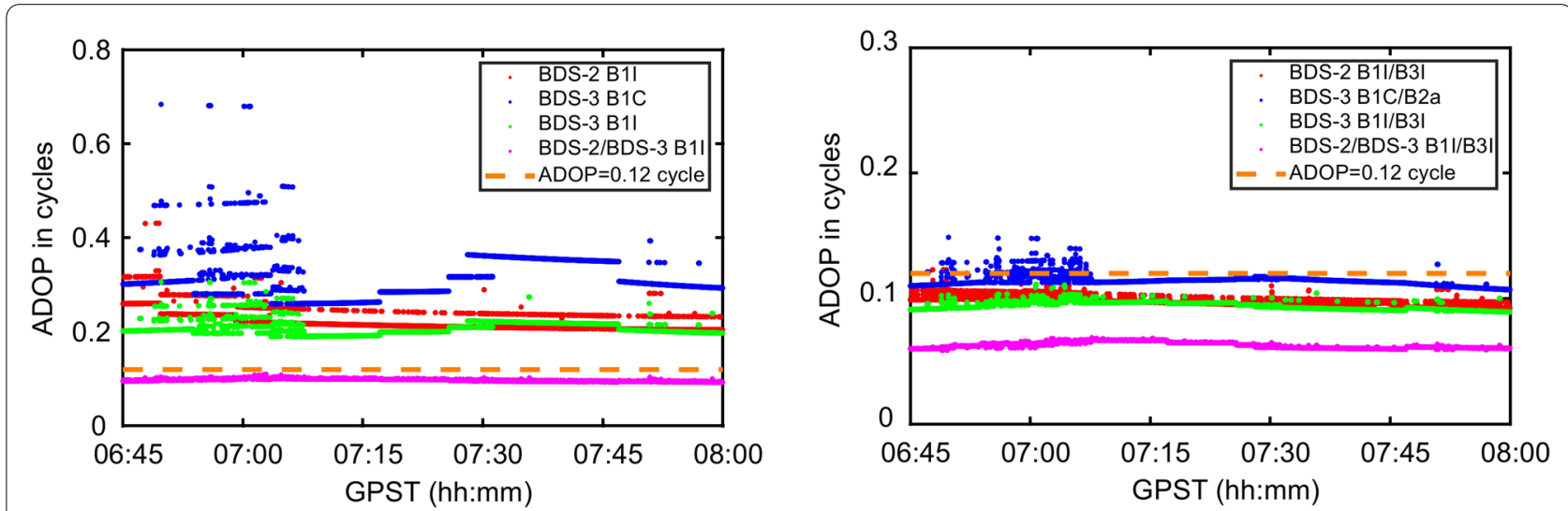

Fig. 15 ADOP based on single-frequency (left) and dual-frequency (right) observations at the elevation cut-off angle of $10^{\circ}$ for BDS-2, BDS-3, and tightly combined BDS-3/BDS-2 solutions

larger than 0.12 cycles, whereas they were smaller than 0.12 cycles for tightly combined BDS-3/BDS-2 solution. Meanwhile, the dual-frequency ADOP values were generally well below 0.12 cycles for BDS-2, BDS-3, and tightly combined BDS-3/BDS-2 solutions. The average ADOP values were $0.228,0.317,0.209$, and 0.098 cycles for BDS-2 B1I, BDS-3 B1C, BDS-3 B1I and tightly combined BDS-3/BDS-2 B1I solutions, respectively, whereas they were $0.099,0.114,0.095$, and 0.063 cycles for the four dual-frequency solutions, respectively.

Figure 16 shows the ambiguity resolution success rates based on single-frequency observations at different elevation cut-off angles. In contrast to the static test, the success rates of the BDS-2 B1I solutions were much lower than those of the BDS-3 B1I solutions at all elevation cutoff angles. The BDS-3 B1I solutions gave the success rates of approximately $62.6-68.5 \%$ with the elevation cut-off angle below $40^{\circ}$ and $51.3 \%$ at the elevation cut-off angle of $40^{\circ}$, whereas the success rates of BDS-2 B1I solutions decreased dramatically from approximately $58.9-5.4 \%$

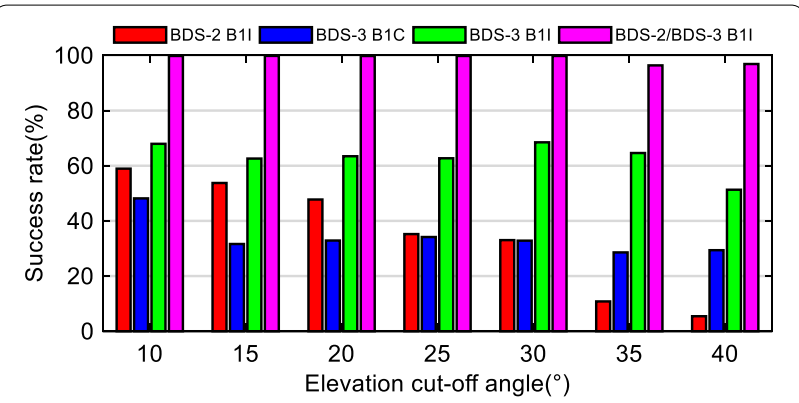

Fig. 16 Single-epoch ambiguity resolution success rates based on single-frequency observations

with increasing elevation cut-off angle from $10^{\circ}$ to $40^{\circ}$. For tightly combined BDS-3/BDS-2 solutions, the success rates of approximately $99.8 \%$ were obtained with elevation cut-off angle below $35^{\circ}$, and approximately $96.4 \%$ and $96.9 \%$ with the elevation cut-off angles of $35^{\circ}$ and $40^{\circ}$, respectively, which again demonstrates the benefits of the tightly combined BDS-3/BDS-2 for precise relative 


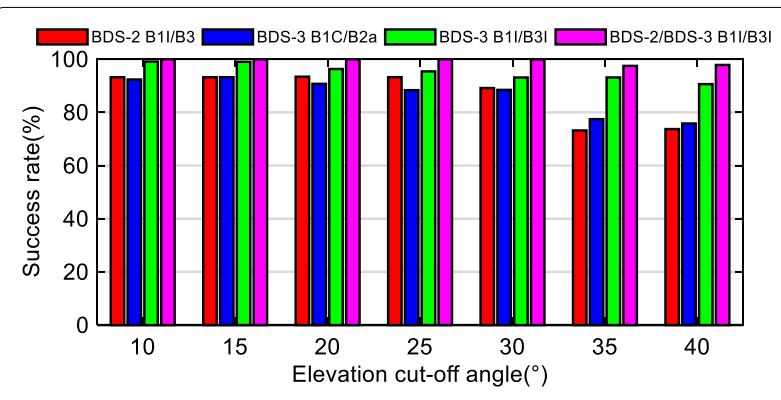

Fig. 17 Single-epoch ambiguity resolution success rates based on dual-frequency observations

positioning. Additionally, compared with BDS-2 B1I solutions, the success rates of BDS-3 B1C solutions were much lower with the elevation cut-off angle below $25^{\circ}$ and much higher beyond the elevation cut-off angle of $30^{\circ}$. It is noteworthy that the success rates for the BDS-3 B1I solutions increased with an increase in the elevation cut-off angle from $25^{\circ}$ to $30^{\circ}$, which was due to the low observational quality of BDS-3 $\mathrm{C} 19$ within a range of the elevation angle from approximately $25^{\circ}$ to $30^{\circ}$.

Figure 17 shows the ambiguity resolution success rates with dual-frequency observations under different elevation cut-off angles. Similarly, the success rates of the BDS-2 B1I/B3I solutions were lower than those of the BDS-3 B1I/B3I solutions at all elevation cut-off angles. However, different from the static test, they were in general comparable to the BDS-3 B1C/B2a solutions. With increasing elevation cut-off angle from $10^{\circ}$ to $40^{\circ}$, the success rates decreased from approximately $99.1-90.6 \%$ for the BDS-3 B1I/B3I solutions. Meanwhile, the success rates of almost $100 \%$ were obtained for the tightly combined BDS-3/BDS-2 solutions at the elevation cut-off angle below $35^{\circ}$, and $97.5 \%$ and $97.8 \%$ under the elevation cut-off angles of $35^{\circ}$ and $40^{\circ}$, respectively. The results demonstrate that a promising RTK performance can be achieved with the dual-frequency observations from the current BDS-3 full constellation alone or the combination of BDS-2 and BDS-3.

Figure 18 depicts the positioning errors in the $E, N$, and $U$ components for BDS-2, BDS-3, and tightly combined BDS-3/BDS-2 based on dual-frequency observations under the elevation cut-off angle of $10^{\circ}$, and the positioning accuracy (only for ambiguity fixed solutions) is listed in Table 7. For the B1I/B3I signals, the positioning accuracy of the BDS-3 solution $(0.52 \mathrm{~cm} / 0.39 \mathrm{~cm} / 2.14 \mathrm{~cm})$ was obviously better than that of the BDS-2 solution $(0.85 \mathrm{~cm} / 1.02 \mathrm{~cm} / 3.01 \mathrm{~cm})$, particularly in the North and Up components. The combined BDS-3/BDS-2 solution improved the positioning accuracy with RMS values of $0.52 \mathrm{~cm} / 0.22 \mathrm{~cm} / 1.80 \mathrm{~cm}$. The 3D position RMS was $3.29 \mathrm{~cm}$ for BDS-2, $2.24 \mathrm{~cm}$ for BDS-3, and $1.89 \mathrm{~cm}$ for

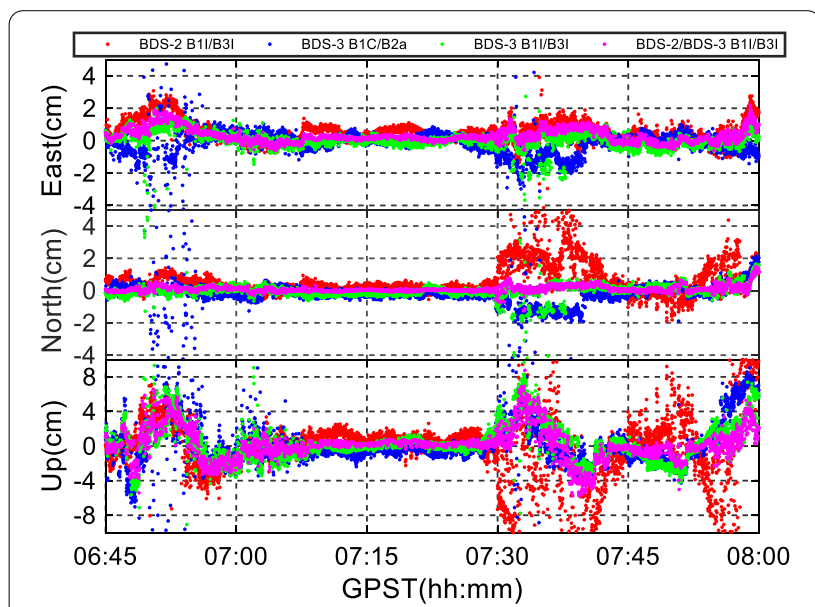

Fig. 18 The positioning errors with dual-frequency BDS-3/BDS-2 observations at the elevation cut-off angle of $10^{\circ}$

tightly combined BDS-3/BDS-2 solution. Moreover, the RMS of BDS-3 B1C/B2a solution were slightly larger than those of the BDS-3 B1I/B3I solution, whereas they were obviously smaller than BDS-2 B1I/B3I solution.

\section{Conclusions and discussions}

In this study, we assessed the single-epoch RTK positioning performance of tightly combined BDS-2 and the newly completed BDS-3 full constellations. We first investigated the existence of DISBs between BDS-3/ BDS-2 B1I/B3I signals. Then we assessed the RTK positioning performance for short baselines with single- and dual-frequency observations from BDS-2/BDS-3 in aspects of ADOP, ambiguity resolution success rate, as well as positioning accuracy. Both static and kinematic datasets collected in Wuhan were processed and analyzed. The following conclusions were obtained:

1. Regarding the specific receivers employed in this study, it was demonstrated that the BDS-3/BDS-2 B1I/B3I code and phase DISBs were approximately zero for baselines with the same or different receiver types at their endpoints, implying that when the legacy B1I/B3I signals were used for precise relative

Table 7 RMS of the single-epoch BDS-2/BDS-3 RTK positioning errors

\begin{tabular}{lllll}
\hline Observations & $\boldsymbol{E}(\mathbf{c m})$ & $\boldsymbol{N}(\mathbf{c m})$ & $\boldsymbol{U}(\mathbf{c m})$ & 3D $(\mathbf{c m})$ \\
\hline BDS-2 B11/B3| & 0.85 & 1.02 & 3.01 & 3.29 \\
BDS-3 B1//B31 & 0.52 & 0.39 & 2.14 & 2.24 \\
BDS-3 B1C/B2a & 0.71 & 0.66 & 2.36 & 2.55 \\
BDS-2/BDS-3 B11/B31 & 0.52 & 0.22 & 1.80 & 1.89 \\
\hline
\end{tabular}


positioning, BDS-3 and BDS-2 can be considered as one constellation without additional DISBs. Therefore, the tightly combined BDS-3/BDS-2 B1I/B3I observations can be easily processed in precise relative positioning. These results implied the full interoperability between BDS-3 and BDS-2 B1I/B3I signals for precise relative positioning.

2. BDS-3 only solution delivered an ambiguity resolution performance comparable to that of the BDS-2 only solution. The performance can be much improved by using a tightly combined BDS-3/BDS-2 solution. Although reliable single-epoch single-frequency ambiguity resolution was infeasible for the BDS-2 only and BDS-3 only solutions, it was feasible for tightly combined BDS-3/BDS-2 solution. The single-epoch single-frequency BDS-3/BDS-2 tightly combined solution can correctly fix the ambiguities with success rate of more than $96.9 \%$ even at the elevation cut-off angle of $40^{\circ}$, indicating a superior performance of the tightly combined BDS-3/BDS-2 RTK in the Asia-Pacific region. About the dual-frequency cases, the ambiguities were successfully resolved with the rates exceeding $90.6 \%$ and $97.8 \%$ at the elevation cut-off angle of $40^{\circ}$ for BDS-3 and tightly combined BDS-3/BDS-2, respectively.

3. The positioning accuracy of the BDS-3 only solution was obviously better than BDS-2 only solution due to better satellite distribution geometry for the current BDS-3 full constellation; additionally, it can be significantly improved by a tight combination of BDS-3 and BDS-2. Our kinematic experiment demonstrated that the RMS of three-dimensional position was $3.29 \mathrm{~cm}$ for BDS-2, 2.24 cm for BDS-3, and $1.89 \mathrm{~cm}$ for tightly combined BDS-3/BDS-2.

4. Because C59 and C60 transmit only B1I/B3I signals but not B1C/B2a signals currently, the ambiguity resolution and positioning performances of BDS-3 B1I and B1I/B3I solutions were much better than those of BDS-3 B1C and B1C/B2a solutions. These results confirmed that the BDS-3 GEO satellites contribute significantly to single-epoch precise relative positioning in the Asia-Pacific region.

Additionally, we computed and compared (but not presented herein) the RTK performance of tightly combined and loosely combined BDS-3/BDS-2 solutions. As expected, the improvements were marginal (less than $1.5 \%)$ for both the single- and dual-frequency cases, which is reasonable considering the facts that the average number of observed satellites was about 13.5, and the ambiguity resolution success rates exceeded $95.6 \%$ for the loosely combined model even under an elevation cut-off angle of $40^{\circ}$. These results indicated that the improvements for the combined BDS-3/BDS-2 solutions were mainly due to the larger number of the observed satellites. Furthermore, we found that the improvements were significant when single-frequency observations were used and the elevation cut-off angle was above $40^{\circ}$, thereby confirming the benefits of the tightly combined BDS-3/BDS-2 solutions in challenging observational environments. It is noteworthy that the benefits of the tightly combined BDS-3/BDS-2 model are more easily experienced outside the Asia-Pacific region, as the number of visible BDS-3/BDS-2 satellites there decreases dramatically in the absence of GEO and IGSO satellites.

It is noteworthy that only 29 among 30 BDS-3 satellites are currently operational. With operation of the last BDS-3 GEO satellite, BDS-3 RTK performance in the Asia-Pacific region will be improved. One should also note that the above results are derived based on the experimental data collected in Wuhan. Future tests should be performed using the data collected from different areas on a global scale. In addition, the existence of DISBs between BDS-3/BDS-2 B1I/B3I signals should be investigated for more types of commercial GNSS receivers.

\section{Abbreviations}

ADOP: Ambiguity dilution of precision; BDS: BeiDou navigation satellite system; BDS-1: BeiDou demonstration navigation satellite system; BDS-2: BeiDou regional navigation satellite system; BDS-3: BeiDou global navigation satellite system; DISB: Differential inter-system bias; GNSS: Global navigation satellite system; Galileo: Galileo navigation satellite system; GPS: Global positioning system; GPST: GPS time; GEO: Geostationary orbit; IGSO: Inclined geostationary orbit; MEO: Medium earth orbit; MGEX: Multi-GNSS experiment; PDOP: Position dilution of precision; RMS: Root-mean-square; RTK: Real-time kinematic; 3D: Three-dimensional.

\section{Acknowledgements}

The authors would like to acknowledge MGEX for providing the GNSS data and broadcast ephemerides. Figure 1 was plotted based on RTKLIB developed by Tomoji Takasu from Tokyo University of Marine Science and Technology, and Figs. $3 \mathrm{a}$ and $12 \mathrm{c}$ were edited using Google Earth. The authors appreciate the constructive and valuable comments from the anonymous reviewers.

\section{Author contributions}

WL and XZ proposed the idea and drafted the article; MW, WW, and WK performed the evaluation and assisted in data analysis; WL and ZZ assisted in data collection; WL and MW assisted in article revision. All authors have read and approved the final manuscript.

\section{Authors' Information}

Wanke Liu is currently a professor at Wuhan University. He obtained his B.Sc., Master, and Ph.D. degrees in Geodesy and Survey Engineering from the School of Geodesy and Geomatics of Wuhan University in 2001, 2004, and 2008, respectively. His main research interests include GNSS precise positioning and multi-sensor integrated positioning. E-mail:wkliu@sgg.whu.edu.cn.

Mingkui Wu is currently a lecturer in the China University of Geosciences (Wuhan). He obtained his B.Sc., and Master, and Ph.D. degrees in Geodesy and Survey Engineering from the School of Geodesy and Geomatics of Wuhan University in 2010, 2012, and 2017, respectively. His main research interests include multi-GNSS precise positioning and GNSS attitude determination. E-mail:wumk@cug.edu.cn 
Xiaohong Zhang is currently a professor in Wuhan University. He obtained his B.Sc., Master, and Ph.D. degrees with distinction in Geodesy and Survey Engineering from the School of Geodesy and Geomatics of Wuhan University in 1997, 1999, and 2002, respectively. His main research interests include precise point positioning and GNSS/INS integration. E-mail: xhzhang@sgg.whu.edu.cn.

Wang Wang is currently a postgraduate in the School of Geodesy and Geomatics, Wuhan University. He obtained his Bachelor degree in surveying and mapping engineering from the School of Geodesy and Geomatics of Wuhan University in 2015. His current research interest is GNSS attitude determination.E-Mail:Wangw158@whu.edu.cn

Wei Ke is currently a postgraduate in the School of Geodesy and Geomatics, Wuhan University. He obtained his Bachelor degree from the College of Civil Engineering of Hefei University of Technology in 2019. His current research interest is multi-GNSS precise relative positioning. E-Mail: keweisgg@whu.edu. $\mathrm{cn}$

Zhiqin Zhu is currently a lecturer in Wuhan University. He obtained his Ph.D. degree in Geodesy and Survey Engineering from the School of Geodesy and Geomatics of Wuhan University in 2012. His main research interests include GNSS/INS integrated navigation and precise GNSS data processing. E-mail: zhqzhu@sgg.whu.edu.cn

\section{Funding}

This research was funded by the National Natural Science Foundation of China (Nos. 41774031, 41904035, 91638203), Hubei Provincial Natural Science Foundation of China (No. 2019CFB261), the National Science Fund for Distinguished Young Scholars (No. 41825009), and Key Laboratory of Geospace Environment and Geodesy, Ministry of Education, Wuhan University (No. 19-01-06).

\section{Availability of data and materials}

The BDS-2/BDS-3 raw observations from MGEX station WUH2 are available at $\mathrm{ftp} / / /$ cddis.gsfc.nasa.gov/pub/gps/data/daily/. All the remaining BDS-2/BDS-3 observations are not publicly available. For more details, please contact the corresponding author by email: xhzhang@sgg.whu.edu.cn.

\section{Competing interests}

The authors declare that they have no competing interests.

\section{Author details}

1 School of Geodesy and Geomatics, Wuhan University, Wuhan 430079, China. ${ }^{2}$ School of Geography and Information Engineering, China University of Geosciences (Wuhan), Wuhan 430074, China.

Received: 24 July 2020 Accepted: 19 January 2021

Published online: 05 April 2021

\section{References}

China Satellite Navigation Office (CSNO). (2019-12). Development of the BeiDou Navigation Satellite System (Version 4.0). http://www.beidou.gov.cn/ xt/gfxz/201912/P020191227430565455478.pdf. Accessed 23 June 2020.

Herring, T. A., King, R. W., Floyd, M. A., \& McClusky, S. C. (2018). GAMIT Reference Manual: GPS Analysis at MIT (Release 10.7). Massachusetts Institute of Technology (MIT). http://geoweb.mit.edu/gg/GAMIT_Ref.pdf. Accessed 30 August 2020.

Hou, P. Y., Zhang, B. C., Yuan, Y. B., Zhang, X., \& Zha, J. P. (2019). Stochastic modeling of BDS2/3 observations with application to RTD/RTK positioning. Measurement Science and Technology, 30(9), 095002.

IGS, \& RTCM-SC104. (2018). RINEX-The receiver independent exchange format Version RINEX 3.04, 23 November 2018. International GNSS Service (IGS), RINEX Working Group and Radio Technical Commission for Maritime Services Special Committee 104 (RTCM-SC104). http://acc.igs. org/misc/rinex304.pdf. Accessed 23 June 2020.

Jiao, G. Q., Song, S. L., \& Jiao, W. H. (2019). Improving BDS-2 and BDS-3 joint precise point positioning with time delay bias estimation. Measurement
Science and Technology, 31(12), 025001. https://doi.org/10.1088/13616501/ab41cf.

Lu, M. Q., Li, W. Y., Yao, Z., \& Cui, X. W. (2019). Overview of BDS III new signals. Navigation, 66(1), 19-35.

Mi, X. L., Zhang, B. C., Yuan, Y. B., \& Luo, X. W. (2020). Characteristics of GPS, BDS2, BDS3 and Galileo inter-system biases and their influence on RTK positioning. Measurement Science and Technology, 31(1), 015009.

Odijk, D., Nadarajah, N., Zaminpardaz, S., \& Teunissen, P. J. G. (2017). GPS, Galileo, QZSS and IRNSS differential ISBs: Estimation and application. GPS Solutions, 21(2), 439-450.

Odijk, D., \& Teunissen, P. J. G. (2008). ADOP in closed form for a hierarchy of multi-frequency single-baseline GNSS models. Journal of Geodesy, 82(8), 473-492.

Odijk, D., \& Teunissen, P. J. G. (2013). Characterization of between-receiver GPS-Galileo inter-system biases and their effect on mixed ambiguity resolution. GPS Solutions, 17(4), 521-533.

Paziewski, J., \& Wielgosz, P. (2015). Accounting for Galileo-GPS inter-system biases in precise satellite positioning. Journal of Geodesy, 89(1), 81-93.

Qu, L. Z., Du, M. Y., Wang, J., Gao, Y., Zhao, Q. L., Zhang, Q., \& Guo, X. (2019). Precise point positioning ambiguity resolution by integrating BDS-3e into BDS-2 and GPS. GPS Solutions, 23(3), 63. https://doi.org/10.1007/s1029 $1-019-0854-y$

Shi, J. B., Ouyang, C. H., Huang, Y. S., \& Peng, W. J. (2020). Assessment of BDS-3 global positioning service: Ephemeris, SPP, PPP, RTK, and new signal. GPS Solutions, 24(3), 81. https://doi.org/10.1007/s10291-020-00995-y.

Song, Z. Y., Chen, J. P., Wang, B., \& Yu, C. (2020). Analysis and modeling of the inter-system bias between BDS-2 and BDS-3. In J. D. Sun, C. F. Yang, \& J. Xie (Eds.), China satellite navigation conference (CSNC) 2020 proceedings (Vol. Il, pp. 279-289). Singapore: Springer.

Test and Assessment Research Center of China Satellite Navigation Office (CSNO-TARC). (2020). Constellation status. http://www.csno-tarc.cn/en/ system/constellation. Accessed 30 June 2020.

Teunissen, P. J. G. (1995). The least-squares ambiguity decorrelation adjustment: A method for fast GPS integer ambiguity estimation. Journal of Geodesy, 70(1-2), 65-82.

Teunissen, P. J. G. (1997). A canonical theory for short GPS baselines. Part IV: Precision versus reliability. Journal of Geodesy, 71(9), 513-525.

Wu, M. K., Liu, W. K., Wang, W., \& Zhang, X. H. (2019a). Differential inter-system biases estimation and initial assessment of instantaneous tightly combined RTK with BDS-3, GPS, and Galileo. Remote Sensing, 11(12), 1430.

Wu, M. K., Zhang, X. H., Liu, W. K., Ni, S. J., \& Yu, S. (2017). Tightly combined BeiDou B2 and Galileo E5b signals for precise relative positioning. The Journal of Navigation, 70(6), 1253-1266.

Wu, M. K., Zhang, X. H., Liu, W. K., Wu, R. P., Zhang, R. L., Le, Y., \& Wu, Y. X. (2019b). Influencing factors of GNSS differential inter-system bias and performance assessment of tightly combined GPS, Galileo, and QZSS relative positioning for short baseline. The Journal of Navigation, 72(4), 965-986.

Xie, X., Fang, R. X., Geng, T., Wang, G. X., Zhao, Q. L., \& Liu, J. N. (2018). Characterization of GNSS signals tracked by the iGMAS network considering recent BDS-3 satellites. Remote Sensing, 10(11), 1736.

Xie, X., Geng, T., Zhao, Q. L., Liu, J. N., \& Wang, B. (2017). Performance of BDS-3: Measurement quality analysis, precise orbit and clock determination. Sensors, 17(6), 1233.

Xu, X. L., Li, M., Li, W. W., \& Liu, J. N. (2018). Performance analysis of BeiDou-2/ BeiDou-3e combined solution with emphasis on precise orbit determination and precise point positioning. Sensors, 18(1), 135.

Xu, X. L., Wang, X. L., Liu, J. N., \& Zhao, Q. L. (2019). Characteristics of BD3 global service satellites: POD, open service signal and atomic clock performance. Remote Sensing, 11(13), 1559.

Yang, X. Y., Huang, G. W., Zhang, Q., Liu, C. C., Wang, L., \& Qin, Z. W. (2019a). Early analysis of precise orbit and clock offset determination for the satellites of the global BeiDou-3 system. Advances in Space Research, 63(3), 1270-1279.

Yang, Y. X., Gao, W. G., Guo, S. R., Mao, Y., \& Yang, Y. F. (2019b). Introduction to BeiDou-3 navigation satellite system. Navigation, 66(1), 7-18.

Yang, Y. X., Mao, Y., \& Sun, B. J. (2020). Basic performance and future developments of BeiDou global navigation satellite system. Satellite Navigation, 1(1), 1. https://doi.org/10.1186/s43020-019-0006-0. 
Yang, Y. X., Xu, Y. Y., Li, J. L., \& Yang, C. (2018). Progress and performance evaluation of BeiDou global navigation satellite system: Data analysis based on BDS-3 demonstration system. Science China Earth Sciences, 61(5), 614-624.

Yuan, Y., \& Zhang, B. (2014). Retrieval of inter-system biases (ISBs) using a network of multi-GNSS receivers. Journal of Global Positioning System, 13(1), 22-29.

Zhang, P. F., Tu, R., Wu, W. J., Liu, J. H., Wang, X. X., \& Zhang, R. (2020a). Initial accuracy and reliability of current BDS-3 precise positioning, velocity estimation, and time transfer (PVT). Advances in Space Research, 65(4), $1225-1234$.

Zhang, R., Tu, R., Liu, J. H., Hong, J., Fan, L. H., Zhang, P. F., \& Lu, X. C. (2018). Impact of BDS-3 experimental satellites to BDS-2: Service area, precise products, precise positioning. Advances in Space Research, 62(4), 829-844.

Zhang, X. H., Li, X., Lu, C. X., Wu, M. K., \& Pan, L. (2019a). A comprehensive analysis of satellite-induced code bias for BDS-3 satellites and signals. Advances in Space Research, 63(9), 2822-2835.

Zhang, X. H., Wu, M. K., Liu, W. K., Li, X. X., Yu, S., Lu, C. X., \&Wickert, J. (2017). Initial assessment of the COMPASS/BeiDou-3: New-generation navigation signals. Journal of Geodesy, 91(10), 1225-1240.

Zhang, Y. Z., Kubo, N., Chen, J. P., Chu, F. Y., Wang, A. H., \& Wang, J. X. (2020b) Apparent clock and TGD biases between BDS-2 and BDS-3. GPS Solutions, 24(1), 27. https://doi.org/10.1007/s10291-019-0933-0.
Zhang, Y. Z., Kubo, N., Chen, J. P., Wang, J. X., \&Wang, H. (2019b). Initial positioning assessment of BDS new satellites and new signals. Remote Sensing, 11(11), 1320.

Zhang, Z. T., Li, B. F., Nie, L. W., Wei, C. X., Jia, S., \& Jiang, S. Q. (2019c). Initial assessment of BeiDou-3 global navigation satellite system: Signal quality, RTK and PPP. GPS Solutions, 23(4), 111. https://doi.org/10.1007/s1029 1-019-0905-4.

Zhao, W., Chen, H., Gao, Y., Jiang, W. P., \& Liu, X. X. (2020). Evaluation of intersystem bias between BDS-2 and BDS-3 satellites and its impact on precise point positioning. Remote Sensing, 12(14), 2185.

Zhou, R. Y., Hu, Z. G., Zhao, Q. L., Li, P. B., Wang, W., He, C. Y., et al. (2018). Elevation-dependent pseudorange variation characteristics analysis for the new-generation BeiDou satellite navigation system. GPS Solutions, 22(3), 60.

\section{Publisher's Note}

Springer Nature remains neutral with regard to jurisdictional claims in published maps and institutional affiliations.

\section{Submit your manuscript to a SpringerOpen ${ }^{\odot}$ journal and benefit from:}

- Convenient online submission

- Rigorous peer review

- Open access: articles freely available online

- High visibility within the field

- Retaining the copyright to your article

Submit your next manuscript at $\gg$ springeropen.com 